\title{
Constraints on Black Hole Growth, Quasar Lifetimes, and Eddington Ratio Distributions From the Sdss Broad-line Quasar Black Hole Mass Function
}

\section{Citation}

Kelly, Brandon C., Marianne Vestergaard, Xiaohui Fan, Philip Hopkins, Lars Hernquist, and Aneta Siemiginowska. 2010. “CONSTRAINTS ON BLACK HOLE GROWTH, QUASAR LIFETIMES, AND EDDINGTON RATIO DISTRIBUTIONS FROM THE SDSS BROAD-LINE QUASAR BLACK HOLE MASS FUNCTION." The Astrophysical Journal 719 (2): 1315-34. https:// doi.org/10.1088/0004-637x/719/2/1315.

\section{Permanent link}

http://nrs.harvard.edu/urn-3:HUL.InstRepos:41381633

\section{Terms of Use}

This article was downloaded from Harvard University's DASH repository, and is made available under the terms and conditions applicable to Open Access Policy Articles, as set forth at http:// nrs.harvard.edu/urn-3:HUL.InstRepos:dash.current.terms-of-use\#OAP

\section{Share Your Story}

The Harvard community has made this article openly available.

Please share how this access benefits you. Submit a story.

Accessibility 
Draft VERsion SEPTEMBer 24, 2018

Preprint typeset using $\mathrm{L}^{A} \mathrm{~T}_{\mathrm{E}} \mathrm{X}$ style emulateapj v. 11/10/09

\title{
CONSTRAINTS ON BLACK HOLE GROWTH, QUASAR LIFETIMES, AND EDDINGTON RATIO DISTRIBUTIONS FROM THE SDSS BROAD LINE QUASAR BLACK HOLE MASS FUNCTION
}

\author{
Brandon C. Kelly ${ }^{1,2,3}$, Marianne Vestergaard ${ }^{4,5}$, Xiaohui Fan ${ }^{5,6}$, Philip Hopkins ${ }^{7}$, Lars Hernquist $^{3}$, Aneta \\ SiEMIGINOWSKA ${ }^{3}$ \\ Draft version September 24, 2018
}

\begin{abstract}
We present an estimate of the black hole mass function (BHMF) of broad line quasars (BLQSOs) that self-consistently corrects for incompleteness and the statistical uncertainty in the mass estimates, based on a sample of 9886 quasars at $1<z<4.5$ drawn from the Sloan Digital Sky Survey. We find evidence for 'cosmic downsizing' of black holes in BLQSOs, where the peak in their number density shifts to higher redshift with increasing black hole mass. The cosmic mass density for black holes seen as BLQSOs peaks at $z \sim 2$. We estimate the completeness of the SDSS as a function of black hole mass and Eddington ratio, and find that at $z>1$ it is highly incomplete at $M_{B H} \lesssim 10^{9} M_{\odot}$ and $L / L_{E d d} \lesssim 0.5$. We estimate a lower limit on the lifetime of a single BLQSO phase to be $t_{B L}>150 \pm 15$ Myr for black holes at $z=1$ with a mass of $M_{B H}=10^{9} M_{\odot}$, and we constrain the maximum mass of a black hole in a BLQSO to be $\sim 3 \times 10^{10} M_{\odot}$. Our estimated distribution of BLQSO Eddington ratios peaks at $L / L_{E d d} \sim 0.05$ and has a dispersion of $\sim 0.4$ dex, implying that most BLQSOs are not radiating at or near the Eddington limit; however the location of the peak is subject to considerable uncertainty. The steep increase in number density of BLQSOs toward lower Eddington ratios is expected if the BLQSO accretion rate monotonically decays with time. Furthermore, our estimated lifetime and Eddington ratio distributions imply that the majority of the most massive black holes spend a significant amount of time growing in an earlier obscured phase, a conclusion which is independent of the unknown obscured fraction. These results are consistent with models for self-regulated black hole growth, at least for massive systems at $z>1$, where the BLQSO phase occurs at the end of a fueling event when black hole feedback unbinds the accreting gas, halting the accretion flow.
\end{abstract}

Subject headings: galaxies: active — galaxies: mass function — galaxies: statistics

\footnotetext{
${ }^{1}$ bckelly@cfa.harvard.edu

2 Hubble Fellow

${ }^{3}$ Harvard-Smithsonian Center for Astrophysics, 60 Garden St, Cambridge, MA 02138

${ }^{4}$ Freja Fellow, Dark Cosmology Centre, The Niels Bohr Institute, University of Copenhagen

${ }^{5}$ Department of Astronomy, University of Arizona, Tucson, AZ 85721

${ }_{7}^{6}$ Max Planck Institute for Astronomy, Heidelberg, Germany

${ }^{7}$ Miller Fellow, Department of Astronomy, University of California, Berkeley, CA
} 


\section{INTRODUCTION}

Understanding how and when supermassive black holes (SMBHs) grow is currently of central importance in extragalactic astronomy. Observations have established correlations between SMBH mass and host galaxy spheroidal properties, such as luminosity (e.g., Kormendy \& Richstone 1995; McLure \& Dunlop 2001, 2002), stellar velocity dispersion ( $M_{B H^{-}} \sigma$ relationship, e.g., Gebhardt et al. 2000; Merritt \& Ferrarese 2001; Tremaine et al.|2002), concentration or Sersic index (e.g., Graham et al. 2001; Graham \& Driver 2007), bulge mass (e.g., Magorrian et al. 1998; Marconi \& Hunt 2003; Häring \& Rix 2004), and binding energy (e.g., Aller \& Richstone 2007; Hopkins et al. 2007c). These correlations imply that the evolution of spheroidal galaxies and the growth of SMBHs are intricately tied together, where black holes grow by accreting gas, possibly fueled by a major merger of two gas-rich galaxies, until feedback energy from the SMBH expels gas and shuts off the accretion process (e.g., Silk \& Rees 1998; Fabian 1999; Begelman \& Nath 2005; Murray et al. 2005; Hopkins et al. 2009b). This 'self-regulated' growth of black holes has been successfully applied in smoothed particle hydrodynamics simulations (Di Matteo et al. 2005; Springel et al. 2005; Johansson et al. 2009), and has motivated numerous models linking the SMBH growth, the quasar phase, and galaxy evolution (e.g., Haehnelt et al.|1998; Kauffmann \& Haehnelt 2000; Haehnelt \& Kauffmann 2000; Wyithe \& Loeb 2003; Volonteri et al.|2003; Cattaneo et al.|2005; Di Matteo et al. 2008; Somerville et al. 2008; Hopkins et al. 2008a; Croton 2009; Sijacki et al. 2009; Booth \& Schave 2009; Shen 2009, and references therein). Within this framework, the broad line quasar 8 phase persists after feedback energy from the black hole 'blows' the gas away (e.g., Hopkins et al. 2005a, $2006 \mathrm{~b})$. The broad line quasar phase is expected to persist until the accretion rate drops low enough to switch to a radiatively inefficient accretion flow (e.g., Churazov et al. 2005; Hopkins et al. 2009a).

While major-mergers of gas-rich galaxies may fuel quasars at high redshift, and grow the most massive SMBHs, alternative fueling mechanisms are likely at lower redshift and fainter luminosities. Mergers alone do not appear to be sufficient to reproduce the number of X-ray faint AGN (e.g., Marulli et al. 2007), and accretion of ambient gas (e.g., Ciotti \& Ostriker 2001; Hopkins \& Hernquist 2006), may fuel these fainter, lower $M_{B H}$ AGN at lower $z$, resulting in an alternative growth mechanism for these SMBHs. Indeed, many AGN are observed to live in late-type galaxies out to $z \approx 1$ (e.g., Guvon et al. 2006; Gabor et al. 2009), and the X-ray luminosity function of AGN hosted by late-type galaxies suggests that fueling by minor interactions or internal instabilities represents a non-negligible contribution to the accretion history of the Universe (Georgakakis et al. 2009). Furthermore, feedback from the SMBH may continue to affect its environment long after its growth through so-called 'radio mode' feedback (Croton et al. 2006; Bower et al. 2006; Sijacki et al. 2007). Observations qualitatitively support a model where the fueling mechanism for black hole growth depends on the mass of the host dark matter halo, but regardless of the fueling mechanism, black hole feedback and accretion follow a similar evolutionary path (e.g., Hickox et al. 2009; Constantin et al. 2009).

Observationally, a significant amount of recent work has utilized the argument of Soltan (1982) to indirectly map the growth of all SMBHs (e.g., Salucci et al. 1999; Yu \& Tremaine 2002; Shankar et al. 2004, 2009; Marconi et al. 2004; Yu \& Lu 2004; Hopkins et al. 2007b; Merloni \& Heinz 2008). Work along this line has used the correlations between $M_{B H}$ and host galaxy spheroidal properties to infer the local distribution of $M_{B H}$ for inactive black holes, which are assumed to be the relics of past AGN activity. The distribution of $M_{B H}$ as a function of redshift is then estimated by stepping backward from the local distribution of $M_{B H}$, employing a continuity equation describing the 'flow' of black hole number density through bins in $M_{B H}$ (e.g., Small \& Blandford 1992). The quasar luminosity function is used as a constraint on the rate of change in the SMBH mass density, because it traces the accretion of matter onto black holes, modulo the accretion efficiency and the bolometric correction. From this work, it has generally been inferred that black hole growth is dominated by periods of near Eddington accretion, with the most massive SMBHs growing first, and that many SMBHs have non-zero spin.

An alternative to the technique of Soltan $(1982)$ for estimating the SMBH mass function has been used by Siemiginowska \& Elvis (1997) and Hatziminaoglou et al. (2001). These authors used a model for thermal-viscous accretion disk instabilities (Siemiginowska et al. 1996) to calculate the expected distribution of luminosity at a given black hole mass. Based on this calculated distribution, they use the quasar luminosity function to constrain the quasar black hole mass function. Siemiginowska \& Elvis (1997) found evidence for black hole 'downsizing', with the peak of the quasar mass function shifting toward lower masses at lower redshift.

Correlations between the SMBH mass, width of the broad emission lines, and luminosity of the quasar continuum have made it possible to estimate $M_{B H}$ for broad line quasars (BLQSOs) (e.g., Vestergaard \& Peterson 2006; Kelly \& Bechtold 2007), albeit with considerable statistical uncertainty of $\sim 0.4$ dex and various systematic effects (e.g., Krolik 2001; Vestergaard \& Peterson 2006; Greene \& Ho 2006; Marconi et al. 2008; Denney et al. 2009). This offers an alternative to estimating SMBH mass functions based on the Soltan (1982) argument, because the mass function may be estimated directly at all redshifts, and because the distribution of quasar emission line widths provides an additional observational constraint on the mass function. Estimates of $M_{B H}$ obtained from the broad emission lines have been used to estimate the distribution of quasar black hole masses and Eddington ratios at a variety of redshifts (e.g., McLure \& Dunlop 2004; Vestergaard|2004; Kollmeier et al.|2006; Netzer \& Trakhtenbrot 2007; Shen et al. 2008; Fine et al. 2008; Kelly et al. 2008; Trump et al. 2009; Labita et al. 2009a).

The BLQSO black hole mass function (BHMF) maps the comoving number density and evolution of active supermassive black holes contained within broad line AGN, and is therefore a complete census of the population of these SMBHs over cosmic time. The BLQSO BHMF is important for a number of reasons, including the following:

\footnotetext{
8 Throughout this work we will use the terms quasar and AGN to refer generically to broad line AGNs. No luminosity difference between the two is assumed, but they are assumed to have broad emission lines along our line of sight.
} 
- At high redshift SMBHs with masses $M_{B H} \sim 10^{9}$ are already in place by $z \sim 6$ (e.g., Fan et al. 2001b; Jiang et al. 2007), and therefore the high- $z$ BLQSO BHMF places important constraints on the formation and growth of seed SMBHs (e.g., Volonteri et al. 2003, 2008; Lodato \& Natarajan 2007).

- If, after a fueling event, the growth of the SMBH persists until it becomes massive enough such that feedback energy begins to unbind the gas, the active SMBH will be seen as a BLQSO shortly before entering quiescence, and its fractional mass growth will not be significant during this time period (Hopkins \& Hernquist 2006). The BLQSO BHMF thus (1) is related to the distribution of spheroidal binding energies in the central regions of the galaxy (Hopkins et al. 2007a, c; ; Younger et al. 2008), and (2) gives the nearly instantaneous increase in the AGN relic SMBH mass density.

- Because mass is a fundamental physical quantity of SMBHs, we can use the BLQSO BHMF to estimate the duty cycle for broad line quasar activity as a function of mass by comparing the number density of all SMBHs with those seen as BLQSOs. The duty cycle can then be converted into an estimate of the lifetime of BLQSO activity, which is of significant importance for understanding the origin of BLQSO activity. This cannot be done using the quasar luminosity function.

- The distribution of luminosities at a given BLQSO SMBH mass depends on the quasar lightcurve Yu \& Lu 2004; Hopkins \& Hernquist 2006; Yu \& Lu 2008; Hopkins \& Hernquist 2009), which is a function of both evolution in the rate at which fuel is supplied to the accretion disk, and the time-dependent behavior of the accretion disk (Siemiginowska \& Elvis 1997; Hatziminaoglou et al. 2001). Thus, understanding the distribution of $L$ at a given $M_{B H}$, or alternatively the distribution of Eddington ratio, gives insight into the BLQSO fueling mechanism and accretion physics.

The BLQSO BHMF therefore provides an important observational constraint on models of SMBH growth, the onset and duration of quasar activity, quasar feedback, and galaxy evolution.

There have been several estimates of the BHMF of BLQSOs calculated directly from the mass estimates derived from the broad emission lines (Wang et al. 2006; Greene \& Ho 2007; Vestergaard et al. 2008; Vestergaard \& Osmer 2009; Kelly et al. 2009b, hereafter KVF09). In particular, Vestergaard \& Osmer (2009) found evidence for cosmic 'downsizing' of black hole mass, in that the most massive SMBHs are more common at high redshift, consistent with previous work on mapping black hole growth (e.g., Marconi et al. 2004; Merloni 2004; Shankar et al. 2004; Merloni \& Heinz 2008), conclusions based on the quasar luminosity function (e.g., Steffen et al. 2003; Ueda et al. 2003; Croom et al. 2004; La Franca et al. 2005; Hasinger et al. 2005; Hopkins et al. 2007b; Silverman et al. 2008) and the local active BHMF (Heckman et al. 2004). However, a major concern with previous estimates of the BLQSO BHMF is uncorrected incompleteness and the additional broadening caused by the statistical errors in mass estimates (Kelly \& Bechtold 2007; Shen et al. 2008, KVF09). Because the massive end of the BLQSO BHMF falls off with increasing $M_{B H}$, the intrinsic uncertainty scatters more sources into higher $M_{B H}$ bins than lower ones, potentially having a significant effect on the estimated number density of the most massive SMBHs. Furthermore, even if a sample is complete in luminosity, it is not necessarily complete in $M_{B H}$, and the completeness in $M_{B H}$ depends on the unknown Eddington ratio distribution. Motivated by these issues, and the fact that the importance of the BLQSO BHMF demands that its determination be statistically rigorous, KVF09 developed a Bayesian statistical technique for estimating the BLQSO BHMF that self-consistently corrects for the incompleteness in $M_{B H}$ and the statistical uncertainties in the estimates of $M_{B H}$. In this work we apply the technique of KVF09 to the SDSS BLQSO sample of Vestergaard et al. (2008) in order to estimate the black hole mass function of SMBHs that reside in BLQSOs, and discuss the implications for $\mathrm{SMBH}$ growth, quasar lifetimes, and Eddington ratio distributions.

\section{THE DATA}

Our sample is drawn from the Sloan Digital Sky Survey (SDSS) Data Release 3 (DR3) quasar sample as presented by Richards et al. (2006) and Vestergaard et al. (2008). Richards et al. (2006) used 15,180 quasars from the SDSS DR3 to determine the quasar optical luminosity function over $0.3<z<5$. Vestergaard et al. (2008) obtained black hole estimates for 14,434 of the quasars presented in Richards et al. (2006) using scaling relationships between the width of the broad emission lines, continuum luminosity, and black hole mass. The details of the sample and fitting process are described in Richards et al. (2006) and Vestergaard et al. (2008). For completeness, we briefly review the spectral modeling used by Vestergaard et al. (2008) to extract the relevant quantities.

Vestergaard et al. (2008) modeled the observed quasar spectra using a power-law continuum, an optical-UV iron line spectrum based on I Zw I (Vestergaard \& Wilkes 2001; Véron-Cetty et al. 2004), a Balmer continuum, and host galaxy template (Bruzual \& Charlot 2003) for objects at $z<0.5$. The continuum luminosities used for the mass estimates are based on the power-law continuum fits. The emission lines used for the mass estimates in this work are $\mathrm{Mg}$ II and $\mathrm{C}$ IV, and were modeled using multiple Gaussian functions so to reproduce the line profile. Contributions from the narrow emission line region were subtracted from $\mathrm{Mg}$ II when appropriate, and line profiles with strong absorption were ignored. The end result of this analysis was a set of emission line FWHM and continuum luminosities, from which black hole mass estimates were calculated.

We have performed a few additional cuts to the sample presented by Vestergaard et al. (2008) before obtaining our final sample. First, we remove the sources located at $z<1$. We do this because the quasar sample contains a significant number of extended sources below $z<1$, and therefore their $i$-band magnitudes sometimes contain a 
significant contribution from the host galaxy (see the discussion in Richards et al. (2006)). This reduces the effective flux limit at $z<1$, creating an artificial second peak in the redshift distribution. While we could attempt to empirically correct the selection function to account for this, we find it easier to simply limit our analysis to $1<z<4.5$. Finally, in order to make our analysis more robust against uncertainty in the selection function, we omit any sources for which the value of the selection function of Richards et al. (2006) is less than 0.01, and force all values of the selection function to be zero that are $<0.01$. After making these cuts, we were left with a sample of 9886 quasars.

\section{THE STATISTICAL MODEL AND DATA ANALYSIS}

We use an expanded version of the statistical model outlined in KVF09 to estimate the BLQSO BHMF. For completeness, we describe the important aspects of the technique developed by KVF09, and the reader is referred to KVF09 for further details regarding the technique and its derivation. Qualitatively, the technique of KVF09 assumes parameteric forms for the BHMF and the distribution of luminosities at a given $M_{B H}$. The average value of the broad line mass estimates at a given $M_{B H}$ is fixed to be consistent with the scaling relationships reported by Vestergaard \& Peterson (2006) and Vestergaard \& Osmer (2009); we assume that these scaling relationships produce unbiased estimates of $M_{B H}$. The BHMF and distribution of $L$ at a given $M_{B H}$, in combination with a selection function, imply an observed distribution of $z, L$, and FWHM. The technique of KVF09 attempts to recover the BHMF and distribution of $L$ at a given $M_{B H}$ by matching the observed distribution of $z, L$, and FWHM (or, equivalently, the mass estimates) that is implied by the model to the actual observed distribution. The posterior probability distribution is used to quantify how well the implied distributions match the observed distributions. As a result, the technique of KVF09 estimates the probability distribution of the BHMF, and of the distribution of $L$ at a given $M_{B H}$, given the observed data set.

\subsection{Model for the Joint Distribution of $M_{B H}, L, \mathrm{FWHM}$, and $z$}

We model the BLQSO BHMF as a mixture of $K$ log-normal distributions:

$$
\phi\left(\log M_{B H}, \log z\right)=N\left(\frac{d V}{d z}\right)^{-1} \sum_{k=1}^{K} \frac{\pi_{k}}{2 \pi\left|\Sigma_{k}\right|^{1 / 2}} \exp \left[-\frac{1}{2}\left(\mathbf{y}-\mu_{k}\right)^{T} \Sigma_{k}^{-1}\left(\mathbf{y}-\mu_{k}\right)\right],
$$

where $\sum_{k=1}^{K} \pi_{k}=1$. In this work we choose $K=4$, based on our previous experience in working with simulated data sets (KVF09), and because we did not notice a significant difference in the results obtained using larger values of $K$. Here, $N$ is the total number of BLQSOs in the Universe that could be seen by an observer on Earth at the time of the survey, $\mathbf{y}=\left(\log M_{B H}, \log z\right), \mu_{k}$ is the 2-element mean vector for the $k^{\text {th }}$ Gaussian functions, $\Sigma_{k}$ is the $2 \times 2$ covariance matrix for the $k^{\text {th }}$ Gaussian function, and $x^{T}$ denotes the transpose of $x$. In addition, we denote $\pi=\left(\pi_{1}, \ldots, \pi_{K}\right), \mu=\left(\mu_{1}, \ldots, \mu_{K}\right)$, and $\Sigma=\left(\Sigma_{1}, \ldots, \Sigma_{K}\right)$. The variance in $\log M_{B H}$ for Gaussian function $k$ is $\sigma_{m, k}^{2}=\Sigma_{11, k}$, the variance in $\log z$ for Gaussian function $k$ is $\sigma_{z, k}^{2}=\Sigma_{22, k}$, and the covariance between $\log M_{B H}$ and $\log z$ for Gaussian function $k$ is $\sigma_{m z, k}=\Sigma_{12, k}$. The parameters for the mass function are $N, \pi, \mu$, and $\Sigma$.

The distribution of luminosity density at a given black hole mass and wavelength $\lambda$ is assumed to also follow a mixture of $J$ log-normal distributions:

$$
p\left(\log L_{\lambda} \mid M_{B H}\right)=\sum_{j=1}^{J} \frac{\gamma_{j}}{\sqrt{2 \pi \sigma_{l, j}^{2}}} \exp \left[-\frac{1}{2}\left(\frac{\log \lambda L_{\lambda}-\alpha_{0, j}-\alpha_{m, j}\left(\log M_{B H}-9\right)}{\sigma_{l, j}}\right)^{2}\right] .
$$

This represents an extension of the model of KVF09, which only used $J=1 \log$-normal distribution. We made this extension to incorporate additional flexiblity in $p\left(L_{\lambda} \mid M_{B H}\right)$, ensuring that the luminosity distribution, and therefore the black hole mass incompleteness correction, is robust to the particular parameteric form. For each log-normal distribution, the parameters for the distribution of $L_{\lambda}$ at a given $M_{B H}$ are $\gamma_{j}, \alpha_{0, j}, \alpha_{m, j}$, and $\sigma_{l, j}$. We used $J=3$ log-normal distributions, as we did not notice a signficant change in the estimated values of $p\left(L_{\lambda} \mid M_{B H}\right)$ when using $J \geq 3$. We further assess the robustness of our assumed form for $p\left(L_{\lambda} \mid M_{B H}\right)$ in $\oint$ 3.3, and show that our results should not be signficantly altered if the true form of $p\left(L_{\lambda} \mid M_{B H}\right)$ is a power-law, as might be expected from some physical models for BLQSO lightcurves.

In our analysis we use the luminosity density at $1350 \AA$ in order to minimize bias at the highest redshifts introduced from extrapolating the power-law continuum beyond the spectral window. At $z \gtrsim 1.8$ the rest frame $\lambda=1350 \AA$ falls within the observed spectral window for the SDSS sources used in this work, while a smaller redshift window is available when using the luminosity densities calculated at other wavelengths. Furthermore, the $z \sim 1$ distributions of bolometric luminosity did not exhibit any significant difference when using the luminosity density at $1350 \AA$, as compared to that calculated using the luminosity density at $\lambda>1350 \AA$, suggesting that significant biases at lower $z$ are not introduced by extrapolating the power-law continuum.

We can connect the parameters in Equation (2) to the distribution of Eddington ratio and bolometric correction. The monochromatic luminosity is related to the Eddington luminosity ratio $\Gamma_{E d}{ }^{9}$ and bolometric correction $C_{\lambda}$ as

$$
\lambda L_{\lambda}=1.3 \times 10^{38} \frac{\Gamma_{E d d}}{C_{\lambda}} \frac{M_{B H}}{M_{\odot}} \quad\left[\operatorname{erg~s}^{-1}\right] .
$$

${ }^{9}$ We will occasionally use $\Gamma_{E d d}$ to denote the Eddington ratio, instead of $L / L_{E d d}$. We do this in certain instances where it is more notationally convenient to do so. 
Therefore, Equation (2) implies that for the $j^{\text {th }} \log$-normal distribution we are assuming that on average $\log \left(\Gamma_{E d d} / C_{\lambda}\right)=$ $\alpha_{0, j}-47.11+\left(\alpha_{m, j}-1\right) \log M_{B H}$, with a Gaussian scatter about this mean value of standard deviation $\sigma_{l, j}$. We do not make any formal attempt to prohibit Equation (2) from allowing values of $L / L_{E d d}>1$, as this would require us to make an assumption about the bolometeric correction. However, as we will show in $\S$ 4.5. our estimate for $p\left(L_{\lambda} \mid M_{B H}\right)$ implies only a negligible fraction of BLQSOs with $L / L_{E d d}>1$, assuming a constant bolometric correction of $C_{1350}=4.3$ (Vestergaard \& Osmer 2009).

The distribution of emission line widths at a given luminosity density and black hole mass is modeled as a log-normal distribution:

$$
\begin{aligned}
& p\left(\log \mathrm{FWHM}_{B L} \mid L_{B L}, M_{B H}\right)= \\
& \frac{1}{\sqrt{2 \pi\left(\sigma_{B L}^{2}+\sigma_{\mathrm{FWHM}}^{2}\right)}} \exp \left\{-\frac{1}{2} \frac{\left(\log \mathrm{FWHM}_{B L}-\beta_{0}^{B L}+1 / 4 \log L_{B L}-1 / 2 \log M_{B H}\right)^{2}}{\sigma_{B L}^{2}+\sigma_{\mathrm{FWHM}}^{2}}\right\} .
\end{aligned}
$$

Here, $\mathrm{FWHM}_{B L}$ is the line width for a particular broad emission line, $L_{B L}$ is the luminosity density used as an estimate for the broad line region size for a particular broad emission line, $\sigma_{\mathrm{FWHM}}$ is the measured uncertainty in $F W H M$ due to measurement error, and $\beta_{0}^{B L}$ and $\sigma_{B L}$ are the parameters for Equation (44) for a particular broad emission line. We do not make any attempt to correct for radiation pressure on the broad emission line clouds (Marconi et al. 2008), as its importance and effects are currently poorly understood (e.g., for a discussion see Vestergaard \& Osmer 2009).

Equation (44) implies that on average FWHM $\propto M_{B H}^{1 / 2} / L_{B L}^{1 / 4}$, or equivalently $M_{B H} \propto L_{B L}^{1 / 2} \mathrm{FWHM}_{B L}^{2}$. Therefore, we can use the results obtained for the broad emission line scaling estimates of $M_{B H}$ to fix $\beta_{0}$. We use the mass scaling relationship for C IV that is presented by Vestergaard \& Peterson (2006), and the relationship for Mg II that is presented by Vestergaard \& Osmer (2009). As noted in KVF09, these scaling relationships imply $\beta_{0}^{\mathrm{MgII}}=10.61$ and $\beta_{0}^{C I V}=11.33$, and we fix $\beta_{0}$ to these values.

In addition, the dispersion in $\mathrm{FWHM}_{B L}$ at a given $L_{B L}$ and $M_{B H}$ can be related to the scatter in the mass estimates based on the scaling relationships. Vestergaard \& Peterson (2006) find the statistical uncertainty in the broad line mass estimates to be 0.36 dex for C IV. Therefore, because FWHM $\propto M_{B H}^{1 / 2}, \sigma_{B L}(\mathrm{CIV}) \approx 0.18$ dex. Likewise, the intrinsic uncertainty in the broad line mass estimate for $\mathrm{Mg}$ II is found to be $\sim 0.4$ dex (Vestergaard et al., in preparation), and therefore $\sigma_{B L}(\mathrm{MgII}) \approx 0.2 \mathrm{dex}$. However, there have been indications from previous analysis of flux limited surveys, which probe higher redshifts and a narrower range in luminosity than that exhibited by the objects with reverberation mapping data, that the intrinsic scatter in the mass estimates may be smaller than $\approx 0.4$ dex (Kollmeier et al. 2006; Shen et al. 2008; Fine et al. 2008; Steinhardt \& Elvis 2010a,b). This may be caused by correlated scatter in the mass estimates with luminosity (e.g., Shen \& Kelly 2010) or redshift, or a dependence on $\sigma_{B L}$ with luminosity or redshift. Both of these possibilities would decrease the dispersion in the scatter in the mass estimates when only probing a smaller range in $L$, or higher redshifts. In addition, Marconi et al. (2008) find a smaller scatter in the masses estimated using $\mathrm{H} \beta$ when one corrects the reverberation mapped masses for radiation pressure. Because of the possibility for a smaller scatter in the mass estimates, we perform our analysis with both $\sigma_{B L}$ fixed to $\approx 0.2 \mathrm{dex}$, and with $\sigma_{B L}$ as a free parameter.

\subsection{The Posterior Distribution and Fitting Technique}

The technique for estimating the BHMF developed by KVF09 takes a Bayesian approach for performing statistical inference, meaning that it calculates the probability distribution of the mass function, given the observed data. All the information regarding the BHMF and the parameters for the statistical model is contained within the posterior probability distribution, which is defined as the probability distribution of the model, given the observed data. KVF09 derived the posterior distribution for the statistical model described in the previous section. Denoting the model parameters for the shape of the BHMF as $\theta=\left(\pi, \mu, \Sigma, \gamma, \alpha_{0}, \alpha_{m}, \sigma_{l}\right)$, the posterior distribution is

$$
p(\theta \mid \mathrm{FWHM}, L, z) \propto p(\theta)[p(I=1 \mid \theta)]^{-n} \prod_{i=1}^{n} p\left(\mathrm{FWHM}_{i}, L_{\lambda, i}, z_{i} \mid \theta\right),
$$

where the number of data points is $n, p(\theta)$ is the prior on $\theta$, and $p(I=1 \mid \theta)$ is the probability as a function of $\theta$ that an BLQSO makes it into the SDSS DR3 catalogue. We note that if the dispersion in the mass estimates, $\sigma_{B L}$, is also treated as a free parameter, then $\theta$ also contains $\sigma_{B L}$. The joint distribution of FWHM, $L$, and $z$, $p\left(\log \mathrm{FWHM}_{i}, \log L_{\lambda, i}, \log z_{i} \mid \theta\right)$, is given by Equations (30)-(40) in KVF09, modified to use the mixture form for $p\left(L_{\lambda} \mid M_{B H}\right)$. We use the the $\mathrm{Mg}$ II line at $1<z<1.6$, both the Mg II and the C IV line at $1.53<z<1.6$, and the $\mathrm{C}$ IV line at $z>1.6$. We do not use $\mathrm{H} \beta$ because we limit our analysis to $z>1$. At $z \sim 0.8 \mathrm{H} \beta$ is shifted into the water vapor bands, which tends to decrease the FWHM accuracy; Mg II is similarly affected at higher redshifts. In addition, we see systematic changes in the Mg II FWHM distribution above a redshift of 1.6, suggesting the presence of biases, which need further investigation (M. Vestergaard et al., in preparation). The posterior distribution for the BHMF normalization, given $\theta$ and $n$, is given by Equation (16) in KVF09.

The inclusion probability as a function of $\theta$ is calculated by averaging the SDSS DR3 selection function over the distribution of $L_{\lambda}$ and $z$ (see Eq.(46)-(49) in KVF09). In order to simplify our analysis we ignore the lower limit of FWHM $>1000 \mathrm{~km} \mathrm{~s}^{-1}$ on the emission line width for the SDSS DR3 sample. The distribution of FWHM for the 
SDSS DR3 falls off before reaching FWHM $=1000 \mathrm{~km} \mathrm{~s}^{-1}$, and it does not appear that imposing the lower limit results in a non-negligible fraction of the BLQSO population being missed. Therefore, any correction for the lower limit in FWHM is negligible, and we ignore it. In this case, the inclusion probability is

$$
\begin{aligned}
p(I=1 \mid \theta)= & \frac{\Omega}{4 \pi} \int_{L_{\lambda}=0}^{\infty} \int_{z=1}^{4.5} \frac{s\left(L_{\lambda}, z\right)}{L_{\lambda} z \ln 10} \times \\
& \sum_{k=1}^{K} \pi_{k}\left[\sum_{j=1}^{J} \gamma_{j} N\left(\log L_{\lambda} \mid \bar{l}_{k j}(z), V_{l, k j}\right)\right] N\left(\log z \mid \mu_{z, k}, \sigma_{z, k}^{2}\right) d z d L_{\lambda} \\
\bar{l}_{k j}(z)= & \alpha_{o, j}+\alpha_{m, j} \mu_{m, k}+\frac{\alpha_{m, j} \sigma_{m z, k}}{\sigma_{z, k}^{2}}\left(\log z-\mu_{z, k}\right) \\
V_{l, k j}= & \alpha_{m, j}^{2} \sigma_{m, k}^{2}\left(1-\rho_{m z, k}^{2}\right)+\sigma_{l, j}^{2} .
\end{aligned}
$$

Here, $\Omega=1622 \mathrm{deg}^{2}$ is the effective sky area of the SDSS DR3 sample (Richards et al. 2006), $s\left(L_{\lambda}, z\right)$ is the SDSS selection function, $N\left(x \mid \mu, \sigma^{2}\right)$ denotes a Normal distribution with mean $\mu$ and variance $\sigma^{2}$, as a function of $x$, $\mu_{m, k}$ and $\mu_{z, k}$ are the mean values of $\log M_{B H}$ and $\log z$ for the $k^{\text {th }}$ Gaussian function, respectively, and $\rho_{m z, k}$ is the correlation between $\log M_{B H}$ and $\log z$ for the $k^{\text {th }}$ Gaussian function. Note that $\bar{l}_{k}(z)$ and $V_{l, k}$ define the mean and variance in $\log L_{\lambda}$ at a given redshift for the $k^{\text {th }}$ Gaussian function. The integral in Equation (6) is over $1<z<4.5$ because we have removed the sources at $z<1$, and there are no useable broad emission lines at $z \gtrsim 4.5$.

Equation (6) is in terms of the selection function with respect to the luminosity density. As mentioned above, we use the luminosity density at $1350 \AA$ in this work. However, Richards et al. $(2006)$ report their selection function in terms of the $i$-band magnitude. We can convert the selection function of Richards et al. (2006) to be in terms of the BLQSO power-law continuum $L_{1350}$ through the equation

$$
s\left(L_{1350}, z\right)=\int_{-\infty}^{\infty} s(i, z) p\left(i \mid L_{1350}, z\right) d i
$$

where $p\left(i \mid L_{1350}, z\right)$ is the distribution of $i$-band magnitude at a given $L_{1350}$ and $z$, and $s(i, z)$ is the SDSS DR3 selection function in terms of $i$ and $z$. We model the distribution of $i$ magnitudes at a given $L_{1350}$ in different redshift bins as a student's $t$ distribution with mean that depends linearly on $\log L_{1350}$ :

$$
p\left(i \mid L_{1350}, z\right)=\frac{\Gamma[(\nu(z)+1) / 2]}{\Gamma(\nu(z) / 2) \sigma_{i}(z) \sqrt{\nu(z) \pi}}\left(1+\frac{1}{\nu(z)}\left(\frac{i-A_{i}(z)-B_{i}(z) \log L_{1350}}{\sigma_{i}(z)}\right)^{2}\right)^{-(\nu(z)+1) / 2}
$$

The student's $t$ distribution converges to the normal distribution for $\nu \rightarrow \infty$; for finite $\nu$ the t-distribution is more heavy tailed than the normal distribution, and we have found it to better describe the distribution of $i$-magnitudes at a given $L_{1350}$ and $z$. The parameters $A_{i}(z), B_{i}(z), \sigma_{i}(z)$, and $\nu(z)$ are fit by maximizing their posterior probability distribution, given the observed set of $i$ magnitudes and $L_{1350}$. The posterior distribution is given by inserting the assumed distributions into Equation (40) of Kelly (2007), and for simplicity we assume a simple flux limit of $i=19.1$ for $z<2.7$ and $i=20.2$ for $z>2.7$ (e.g., Richards et al. 2006). At $z<2.7$ the redshift bins used in Equation (10) have width $\Delta z=0.1$, while at $z>2.7$ the redshift bins have width $\Delta z=0.3$.

In this work we constrain the parameters of our statistical model to be within certain limits, but in general assume a uniform prior on $\theta$. Our prior is uniform on the parameters within the limits $44.5<\alpha_{0}<46.5,-1<\alpha_{m}<3,0.1<$ $\sigma_{l}<2,7<\mu_{m, k}<10, \log 1<\mu_{z, k}<\log 4.5,0.1<\sigma_{m, k}, \sigma_{z, k}<2$, and $-0.98<\rho_{m z, k}<0.98$. We also assume a uniform prior on $\pi$ and $\gamma$, subject to the elements of $\pi$ and $\gamma$ summing to unity. The limits on $\alpha_{m}$ were chosen because we did not consider it realistic that $L_{1350}$ would depend on $M_{B H}$ outside of the range of dependencies spanning $L_{1350} \propto 1 / M_{B H}$ to $L_{1350} \propto M_{B H}^{3}$, and the limits on $\alpha_{0}$ were chosen to restrict the average value of $L / L_{E d d}$ to be within $0.01<L / L_{E d d}<1$ for BLQSOs with $M_{B H}=10^{9} M_{\odot}$, assuming a bolometeric correction of $C_{1350}=4.3$. The limits on $\sigma_{l}$ were chosen because $\Delta \log L \approx 0.1$ is comparable to the grid spacing on which the selection function was computed by Richards et al. (2006), and we did not consider it likely that the dispersion in $L_{1350}$ at a given $M_{B H}$ would be greater than 2 dex. The limits on the BHMF parameters were chosen so as not to extrapolate the BHMF very far beyond the detectable range of $M_{B H}$. As such, in this work we limit our analysis of the $\mathrm{BHMF}$ to $M_{B H} \gtrsim 10^{7} M_{\odot}$.

As mentioned before, there exists the possibility that, in the range of $L$ and $z$ we probe, the uncertainty in the mass estimates may be smaller than the commonly quoted $\sim 0.4$ dex. If the error in the mass estimates is correlated with luminosity, then the variance in the mass estimates at a given luminosity and mass is reduced to

$$
\operatorname{Var}\left(\log \hat{M}_{B L} \mid L\right)=\operatorname{Var}\left(\log \hat{M}_{B L}\right)\left(1-\rho_{B L}^{2}\right)
$$

Here, $\hat{M}_{B L}$ denotes the broad line mass estimate, $\operatorname{Var}\left(\log \hat{M}_{B L}\right)$ is the variance in the mass estimates about the true mass, typically thought to be $\sim 0.4^{2} \mathrm{dex}^{2}$, and $\rho_{B L}$ is the correlation with luminosity in the scatter in the mass estimates about the true mass. Because we probe a somewhat narrow range in $L_{\lambda}$, when we estimate the parameter 
$\sigma_{B L}$ in Equation (4), we are really estimating $2 \sigma_{B L} \approx \sqrt{\operatorname{Var}\left(\log \hat{M}_{B L} \mid L\right)}$, and we therefore need to also construct a prior for $\sigma_{B L}$. We do this by first noting that Vestergaard \& Peterson (2006) estimated the dispersion in the scatter in the broad line mass estimates about the reverberation mapping estimates using 27 AGN. Therefore, the appropriate prior distribution for $\operatorname{Var}\left(\log \hat{M}_{B L}\right)$ is the posterior probability distribution of the variance in the mass estimates, given the reverberation mapping sample. Since we assume that the scatter in the mass estimates about the true mass is log-normal, the probability distribution of their variance follows using a standard result from Bayesian statistics, and is a scaled inverse $\chi^{2}$ distribution with 26 degrees of freedom (e.g., Gelman et al. 2004). However, there are currently no constraints on the value of $\rho_{B L}$, and we use a uniform prior on its value. Our prior on $\sigma_{B L}$ is then calculated by combining these two priors according to Equation (11). This results in a broad prior which peaks at $\operatorname{Var}\left(\log \hat{M}_{B L} \mid L\right) \approx 0.4^{2} \mathrm{dex}^{2}$, and falls off slowly to zero as $\operatorname{Var}\left(\log \hat{M}_{B L} \mid L\right) \rightarrow 0$ and $\operatorname{Var}\left(\log \hat{M}_{B L} \mid L\right) \rightarrow 0.6^{2}$.

In addition to the above constraints, we also impose the prior constraint that the number density of BLQSO SMBHs must never exceed the local number density of all SMBHs. In principle, this constraint could be violated if a large number of 'wandering' black holes are present. These wandering black holes would be ejected from galactic nuclei in the late stages of a merger due to asymmetric emission of graviational radiation (e.g., Volonteri 2007). However, this constraint would only be violated if the binary black hole system is ejected after or during the broad line quasar phase. While this would certainly be a very intriguing result, we do not test it here; indeed, our results are unaffected by this prior constraint as the estimated BHMF is always below the local value for all random draws from the posterior probability distribution.

As described in KVF09, we do not work with Equation (5) directly, but instead use a Markov Chain Monte Carlo (MCMC) sampler algorithm to obtain a set of random draws of $\theta$, distributed according to Equation (5). Because the posterior distribution described by Equation (5) is multimodal due to ambiguities in labeling the Gaussian functions, we label the BHMF Gaussian functions in order of increasing implied mean flux, and the $p\left(L \mid M_{B H}\right)$ Gaussian functions in order of increasing mean luminosity at $M_{B H}=10^{9} M_{\odot}$. In addition, in order to make our MCMC sampling algorithm robust against additional possible multimodality, we include parallel tempering (e.g., Liu 2004) in our Markov Chain Monte Carlo (MCMC) algorithm to facilitate sampling from the different modes. For each value of $\theta$ we obtained from our MCMC random number generator, we also obtain a value of the BHMF normalization, $N$, by drawing from a negative binomial distribution with parameters $n$ and $p(I=1 \mid \theta)$ (KVF09). The random realizations of $\theta$ and $N$ then define a sample of random realizations of the BHMF obtained from the posterior probability distribution of the BHMF, given the observed set of mass estimates, luminosity densities, and redshifts. We ran our MCMC sampler for $2 \times 10^{5}$ iterations each, keeping every $20^{\text {th }}$ iteration.

\subsection{Robustness to Incorrect Assumptions Regarding the BLQSO BHMF and Eddington Ratio Distribution}

Although we have assumed a flexible parameteric form for the BLQSO BHMF and $p\left(L \mid M_{B H}\right)$, this form is inconsistent with more physically-motivated BLQSO $p\left(L \mid M_{B H}\right)$, such as might be expected from a power-law decay in the BLQSO accretion rate (e.g., Yu et al. 2005; Hopkins \& Hernquist 2006, also see discussion in $\S$ 5.1). Instead, our assumed log-normal mixture form for $p\left(L \mid M_{B H}\right)$ was motivated by mathematical convenience, as some of the integrals necessary for evaluation of the posterior distribution can be done analytically (KVF09). If we were to use a form which required numerical integration, we would have to perform over ten billion numerical integrals in our MCMC sampler, which is computationally prohibitive. Moreover, we also used the mixture form to allow flexibility in the estimated $p\left(L \mid M_{B H}\right)$, so that our assumed form should be able to approximate many different forms for $p\left(L \mid M_{B H}\right)$.

In order to assess the impact of our chosen parameteric form on the inferred BHMF, we simulated a data set where the distribution of Eddington ratios was assumed to have a power-law form $p\left(\Gamma_{E d d} \mid M_{B H}\right) \propto \Gamma_{E d d}^{-(1+1 / \beta)}$ with $\beta=2$ (see discussion in $\S$ 5.1). The distribution of bolometric corrections was log-normal with geometric mean $C_{1350}=5$ and dispersion of 0.2 dex, and the BHMF normalization was $N=2 \times 10^{6}$; all other aspects of the simulation were done in the same manner as described in $\S 6.1$ of KVF09.

The results are illustrated in Figure1, where we compare the true and estimated BHMF for the simulated sample at $z=2$, and the true Eddington ratio distribution with that inferred assuming the statistical model described in $\S 3$.1. Here, and elsewhere in this paper, in addition to a single 'best-fit' mass function, we also plot 100 random draws of the mass function from its probability distribution, generated by our MCMC random number generator. The spread and density of the random draws of the BHMF, and any quantities derived from it, give a visual representation of the uncertainty in these quantities, with the spread on the random draws constraining the BHMF. Because we use 100 random draws, the probability of a quantity falling within a certain area on a plot can be estimated by counting the number of random draws that intersect that area. For this example, the BLQSO BHMF inferred assuming a mixture of $J=3$ log-normal distributions for $p\left(L_{\lambda} \mid M_{B H}\right)$ is able to recover the true BHMF and Eddington ratio distributions, at least when the true distribution of $L / L_{E d d}$ is $p\left(\Gamma_{E d d}\right) \propto \Gamma_{E d d}^{-1.5}$. Although this test is far from exhaustive, we consider it reasonable to conclude that our flexible form for the BHMF and $p\left(L_{\lambda} \mid M_{B H}\right)$ is able to accurately approximate the true forms, and therefore our results are robust against errors resulting from using an incorrect parameteric form for these distributions.

\section{RESULTS}

\subsection{Evaluating the Fit: How Uncertain are the Mass Estimates?}



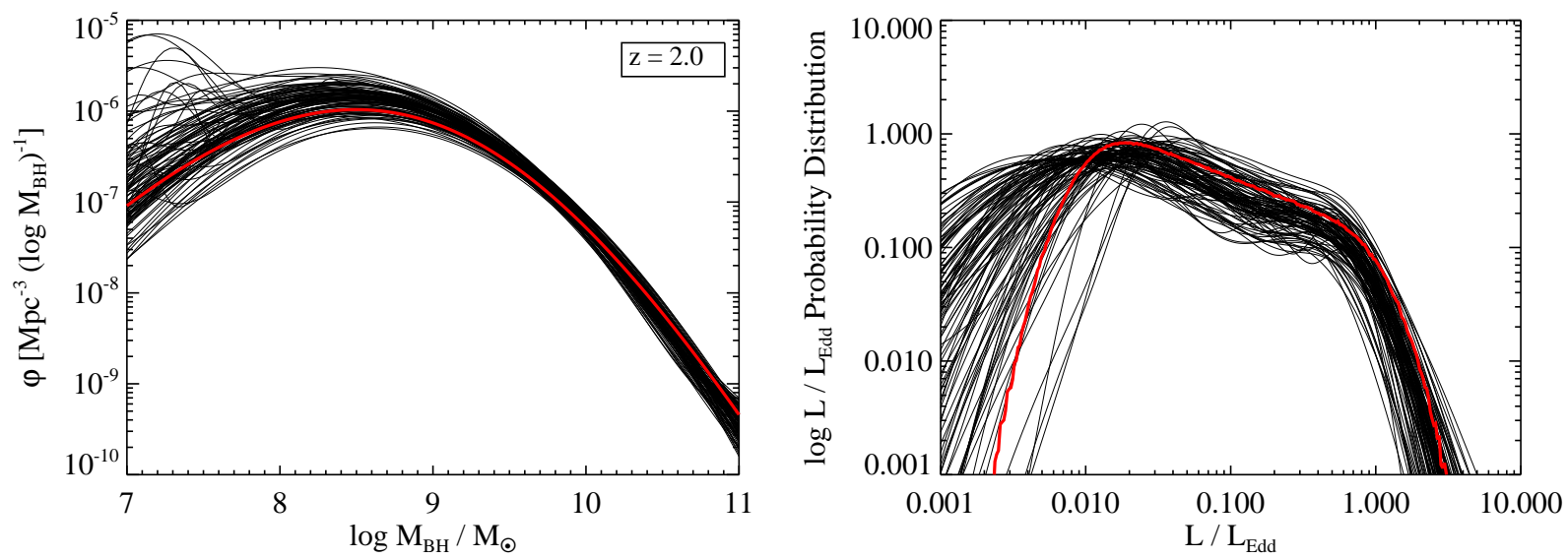

FIG. 1.- (a) True BHMF for the simulated sample described in $\S 3.3$ having a power-law distribution of Eddington ratios over $0.01<$ $L / L_{E d d}<1$ (thick red line), compared with the BHMF estimated assuming a mixture of $J=3$ log-normal distributions of Eddington ratios (thin black lines); each of the thin solid lines denotes a random draw from the probability distribution of the BHMF, given the observed data and assumptions outlined in $\S 3.1$ Here, and in all figures in this work, we plot 100 random draws of the function of interest, so the probability that the BHMF, say, has a certain value in a given range can be estimated by counting the number of random draws of the BHMF that fall within that range. For this example, the BHMF estimated assuming a mixture of log-normal distributions for $L / L_{E d d}$ is able to recover the true BHMF, implying that our mixture form is robust against mispecification of the parameteric model. (b) True Eddington ratio distribution for the same simulated sample (thick red line), compared with the estimated distribution assuming a mixture of $J=3$ log-normal distributions (thin black lines). In this plot we have convolved the power-law form of the distribution of $L / L_{E d d}$ with the scatter in the bolometeric correction used in this simulation, to incorporate the error in the estimated Eddington ration distribution introduced from assuming a constant bolometeric correction. The mixture of log-normals form is able to adequately approximate the true distribution of $L / L_{E d d}$.
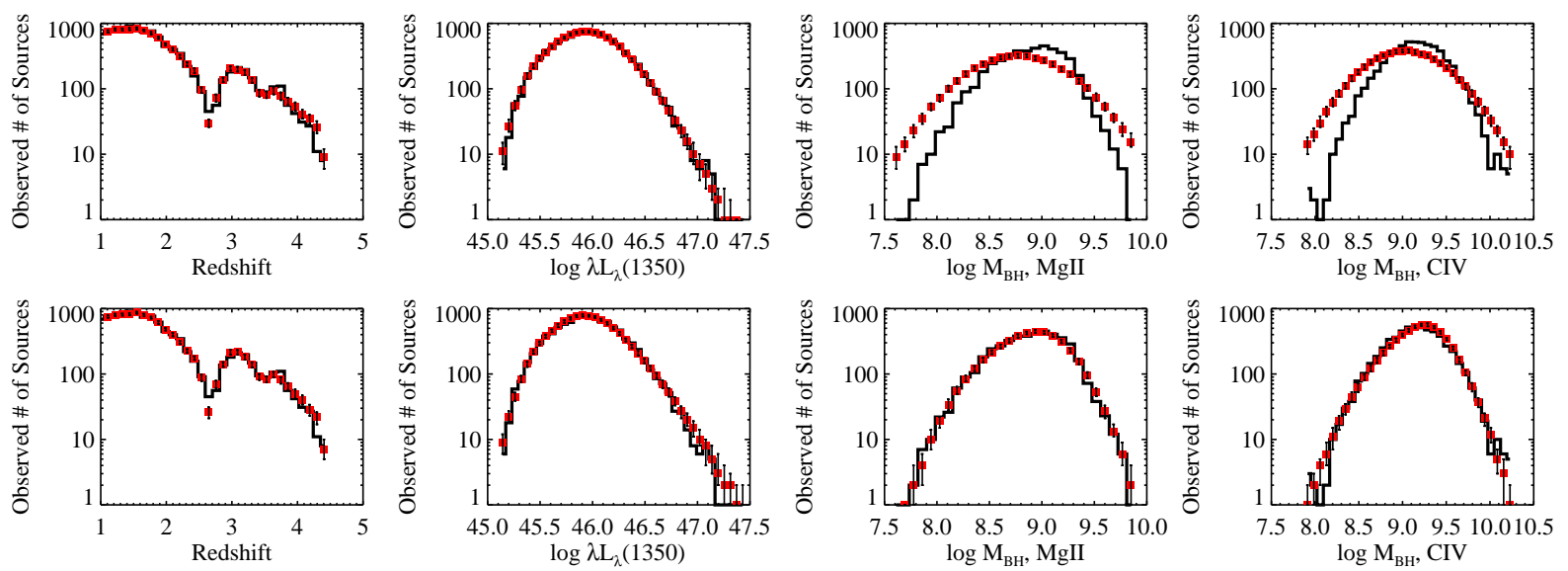

FIG. 2.- Distribution of $z, L_{1350}$, and the mass estimates for our sample (histograms), compared with the distributions implied by our best-fit BHMF and distribution of $L_{1350}$ at a given $M_{B H}$ (red squares). In the top row of plots we fix the statistical error in the mass estimates to be $0.4 \mathrm{dex}$, while in the bottom row we allow it to be a free parameter. The error bars denote the $1 \sigma$ uncertainties on the implied distributions, although often they are smaller than the red squares. The statistical model described in $\delta 3.1$ is able to reproduce the observed distributions only if we allow the standard deviation in the statistical error in the mass estimates to be a free parameter, implying that the standard value of $\sim 0.4$ dex is too large for this luminosity and redshift range.

We used our Bayesian method to derive the BLQSO BHMF from the SDSS DR3 quasar sample, both holding the magnitude of the scatter in mass estimates fixed to 0.4 dex, and treating the amplitude of the scatter as a free parameter. However, before discussing the results, we first evaluate how well the statistical model described in $\S 3.1$ fits our sample. In order to do this, we compare the distributions of redshift, luminosity, and broad line mass estimates of our sample to the distributions implied by our model, as described in KVF09. Figure 2 compares the observed distributions of $z, \lambda L_{\lambda}(1350 \AA)$, and $\hat{M}_{B L}$ to those implied by the statistical model described in $\S 3.1$ The implied distributions were calculated by first simulating a sample of $M_{B H}$ and $z$ from a BHMF randomly output from the MCMC sampler. Then, for each value of $M_{B H}$, we simulated a value of $\lambda L_{\lambda}(1350 \AA)$ and mass estimate $\hat{M}_{B L}$. Finally, we applied the selection function given by Equation (9) to the simulated data set. This was repeated for each realization of the BHMF obtained from the MCMC output, in order to account for the uncertainty in our estimated model parameters.

We are not able to fit the mass estimate distributions if we keep the statistical scatter in the mass estimates fixed to 0.4 dex. In particular, this predicts a distribution of mass estimates that is too broad compared to the actual 
distribution. In fact, the observed dispersion in the mass estimates for our SDSS sample is $\approx 0.35$ dex, smaller than that expected even if all objects had the same mass. However, if we allow the dispersion in the mass estimate error to be a free parameter, we are able to obtain a good match to the data. Our best-fit value for the scatter in the mass estimates at a given mass is $0.18 \pm 0.01$ dex for $\mathrm{Mg}$ II and $0.13 \pm 0.01$ for $\mathrm{C} \mathrm{IV}$. Our result that the scatter in the mass estimates for high $L$ and $z$ must be smaller than $\sim 0.4$ dex is consistent with what has been found in previous work (Kollmeier et al. 2006; Shen et al. 2008; Fine et al. 2008), and our best-fit values of the standard deviations in the mass estimate errors are consistent with the upper limits recently calculated by Steinhardt \& Elvis (2010b).

The origin of this smaller scatter is unclear, and there may be several different possibilities. One possibility, as mentioned earlier and by Shen et al. (2008) and Shen \& Kelly (2010), is that the error in the mass estimates may be correlated with luminosity. If this is true, then an error of $\sim 0.4$ dex represents the error in the mass estimates when averaging over a broad range in $L$, as was done for the reverberation mapping sample, while a smaller scatter of $\sim 0.15$ dex represents the error in the mass estimates when one is limited to a more narrow range in $L$, as the SDSS quasar sample is. It is unclear why the error in the mass estimates would be correlated with luminosity, but one possible source unaccounted for is radiation pressure. Marconi et al. (2008) argue that virial mass estimates should be corrected for radiation pressure. They find a correction that implies a steeper dependence on luminosity than $\hat{M}_{B L} \propto L^{0.5}$, especially for sources with high $L / L_{E d d}$. Under their model, if one does not correct for radiation pressure then one will tend to underestimate the mass with increasing $L$, producing a correlation between the error in the mass estimates with luminosity, and possibly producing a smaller scatter in the mass estimate errors over a narrow range in luminosity. It is interesting to note that Marconi et al. (2008) find a smaller scatter in the mass estimates of $\sim 0.2$ dex when correcting for radiation pressure for the reverberation mapped sample, which covers a larger range in luminosity. However, more work is need to understand the importance of radiation pressure, and if it can produce the observed smaller scatter in the mass estimates over the range in luminosity we probe.

Another possibility is that the error in the mass estimates may not be correlated with $L$, but the dispersion in the errors may decrease with increasing $L$ or $z$. Unfortunately, the number of AGN with $M_{B H}$ estimated from reverberation mapping is too small to test this, and dominated by sources at lower $L$ and $z$. The third possibility is that the virial mass estimates are biased at high $L$ and $z$, at least for Mg II and C IV, and are only marginally related to the actual masses. The $R-L$ relationship is well established for $\mathrm{H} \beta$, and does not require a large extrapolation to the luminosities probed in our sample, and thus we do not expect $\mathrm{H} \beta$-based mass estimates to be significantly biased in this range (Vestergaard 2009). The situation is less clear for Mg II and C IV. There is only one reliable time lag for the Mg II emission line (Metzroth et al.|2006). An $R-L$ relationship has been estimated for the C IV line over a broad range in luminosity and redshift (Kaspi et al. 2007), including those probed in our study, and the $R-L$ relationship is similar for both $\mathrm{H} \beta$ and C IV. Unfortunately, the C IV $R-L$ relationship is estimated from only eight data points, and further work is needed in order to understand the Mg II- and C IV-based mass estimates and their errors.

Throughout the rest of this work will we focus on the results obtained from allowing the dispersion in the mass estimate error to be a free parameter, a value of $\sim 0.4$ dex is clearly ruled out. However, we note that we have performed the same analysis for both cases, and while the quantitative details change, the scientific conclusions are unaffected by treating the amplitude of the scatter as a free parameter. The only exception is that we infer a much more narrow distribution of Eddington ratio if we fix the amplitude of the scatter in the mass estimates to be $\sim 0.4$ dex. In addition, the results reported in this section highlight the need for more reverberation mapping studies in order to better understand the nature of the errors in the mass estimates.

\subsection{The Black Hole Mass Function for Broad Line AGN}

Figure 3 shows the BLQSO BHMF at several redshifts. Our estimated BHMF is compared with an estimate of the local mass function of all SMBHs, and the BHMF reported by Vestergaard et al. (2008), obtained from binning up the broad line mass estimates. Following Merloni \& Heinz (2008), the local BHMF was computed to be near the middle of the uncertainty range reported by Shankar et al. (2009) by convolving a Schechter function with a log-normal distribution with standard deviation 0.3 dex, chosen to be consistent with the scatter about the $M_{B H^{-}} \sigma$ relationship; the parameters for the Schechter function are those reported by Merloni \& Heinz (2008). Also, we note that early type galaxies dominate the local BHMF at $M_{B H} \gtrsim 4 \times 10^{7} M_{\odot}$ (Yu \& Lu 2008).

In order to focus on the region of the BHMF that is robust against uncertainties in the selection function, as well as against uncertainty on the Eddington ratio distribution, we estimate the black hole mass completeness for our sample as a function of $z$. Our best estimate of the SDSS completeness as a function of $M_{B H}$ and $z$ is shown in Figure 4 . and the $10 \%$ completeness limit is marked by a vertical line in Figure 3 . The black hole mass completeness depends on both the completeness in luminosity, and the assumed distribution of $L$ at a given $M_{B H}$. As can be seen from Figure 4 at $z \gtrsim 2$ the SDSS quasar sample is only $\approx 10 \%$ complete at $M_{B H} \sim 10^{9} M_{\odot}$, becoming more incomplete at lower masses. At masses much lower than $M_{B H} \sim 10^{9} M_{\odot}$, the estimated mass function almost completely depends on extrapolation from the set of BHMFs and Eddington ratio distributions that fit the observed data well, constrained by our assumed parameteric forms. Therefore, we stress that below $M_{B H} \sim 10^{9} M_{\odot}$ the BLQSO BHMF must be interpreted with caution, and in this work we will try to focus on what we can infer from the high mass end of the mass function.

We also estimate the completeness of our sample as a function of $L / L_{E d d}$, assuming a constant bolometric correction of $C_{1350}=4.3$. The estimated completeness depends on the selection function and the estimated BHMF, as the selection function depends on luminosity, which is defined by the BHMF at a given $L / L_{E d d}$. The Eddington ratio completeness 


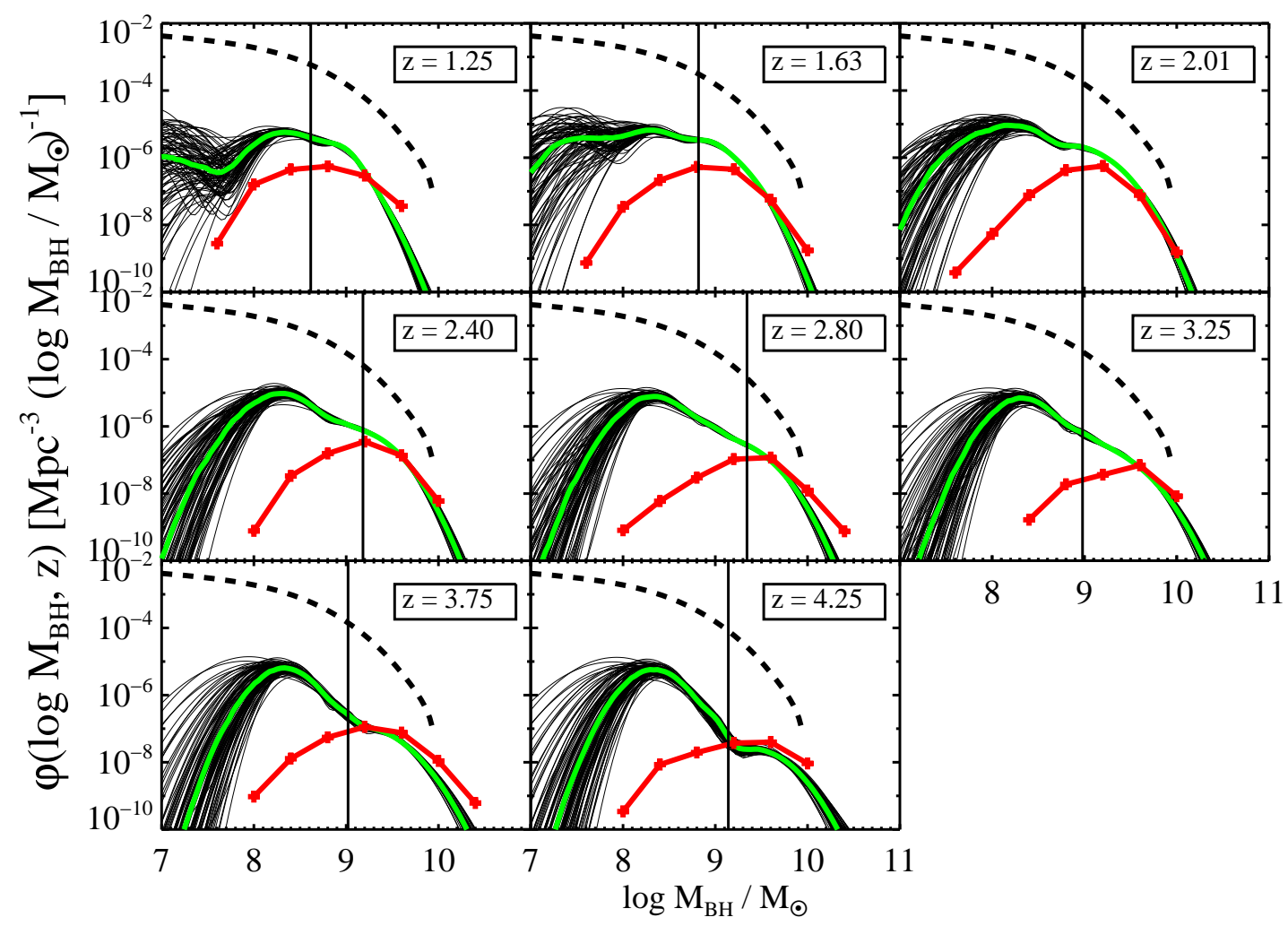

FIG. 3.- BLQSO BHMF (thin solid lines) obtained using our Bayesian approach, compared with the local BHMF fo all SMBHs (dashed line), and the BHMF from Vestergaard et al. (2008, solid red line with points); as in Figure 1 each thin solid line denotes a random draw of the BHMF from its probability distribution. The thick green line is the median of the BHMF random draws, and may be considered our 'best-fit' estimate. The vertical line marks the mass at which the SDSS DR3 sample becomes $10 \%$ complete.
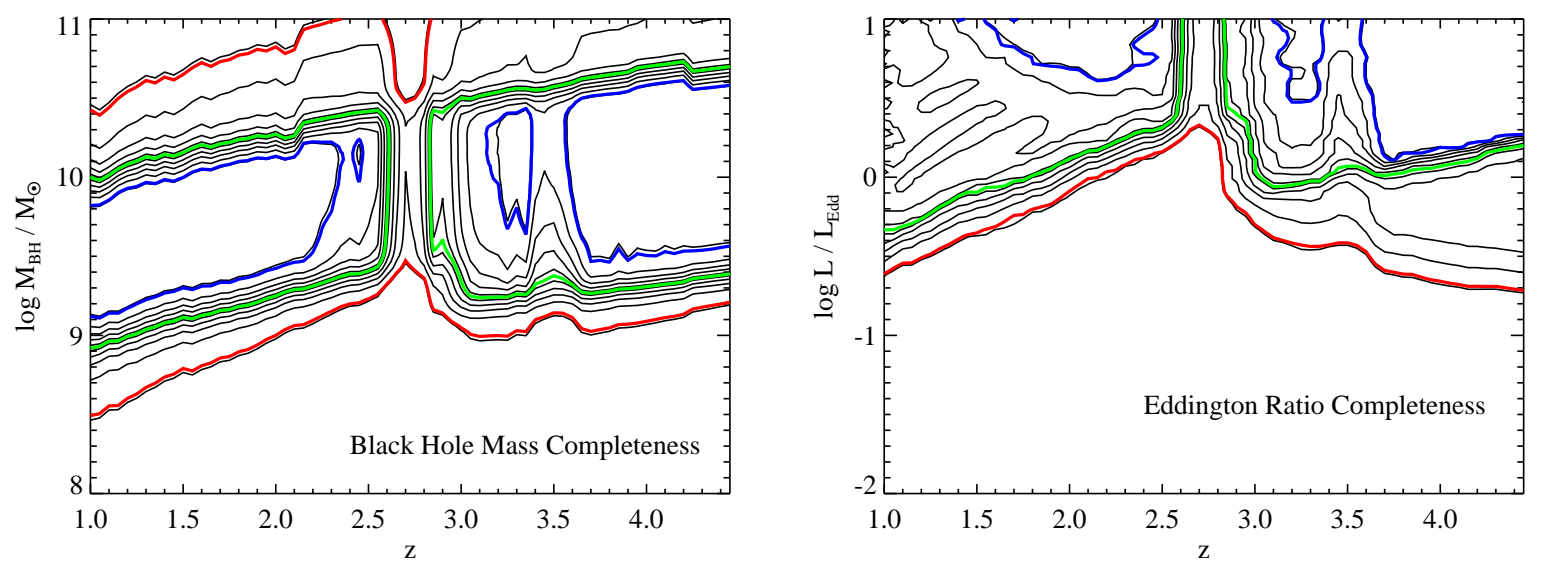

FIG. 4.- Estimated completeness in black hole mass (left) and Eddington ratio (right) for the SDSS DR3 quasar sample of Richards et al. (2006), calculated using our assumed distribution of luminosity at a given black hole mass (Eq. 2). The red, green, and blue lines denote the $10 \%, 50 \%$, and $90 \%$ completeness levels, respectively. The SDSS sample is highly incomplete at $M_{B H} \lesssim 10^{9} M_{\odot}$ and $L / L_{E d d} \lesssim 0.5$. Note that the incompleteness at the highest masses is due to the upper flux limit of the SDSS.

is also shown in Figure 4 The SDSS is only $\sim 50 \%$ complete for sources radiating at the Eddington limit, and $\lesssim 10 \%$ complete for sources radiating at $\lesssim 10 \%$ of Eddington. This heavy incompleteness is due to the fact that, for our estimated BLQSO BHMF, half of BLQSOs have black holes that are not massive enough to make the SDSS flux limit even if they radiate at Eddington. We further discuss the Eddington ratio distribution in Section $\S 4.5$.

The difference between the binned estimate of the BLQSO BHMF, calculated from the estimate of Vestergaard et al. (2008), and our estimate, is the result of the difference in statistical methodology employed by Vestergaard et al. (2008) and our work. First, the statistical error in the broad line mass estimates results in a broader inferred BHMF when one simply bins up these estimates (e.g., Kelly \& Bechtold 2007, KVF09). Second, the $1 / V_{a}$ technique corrects 

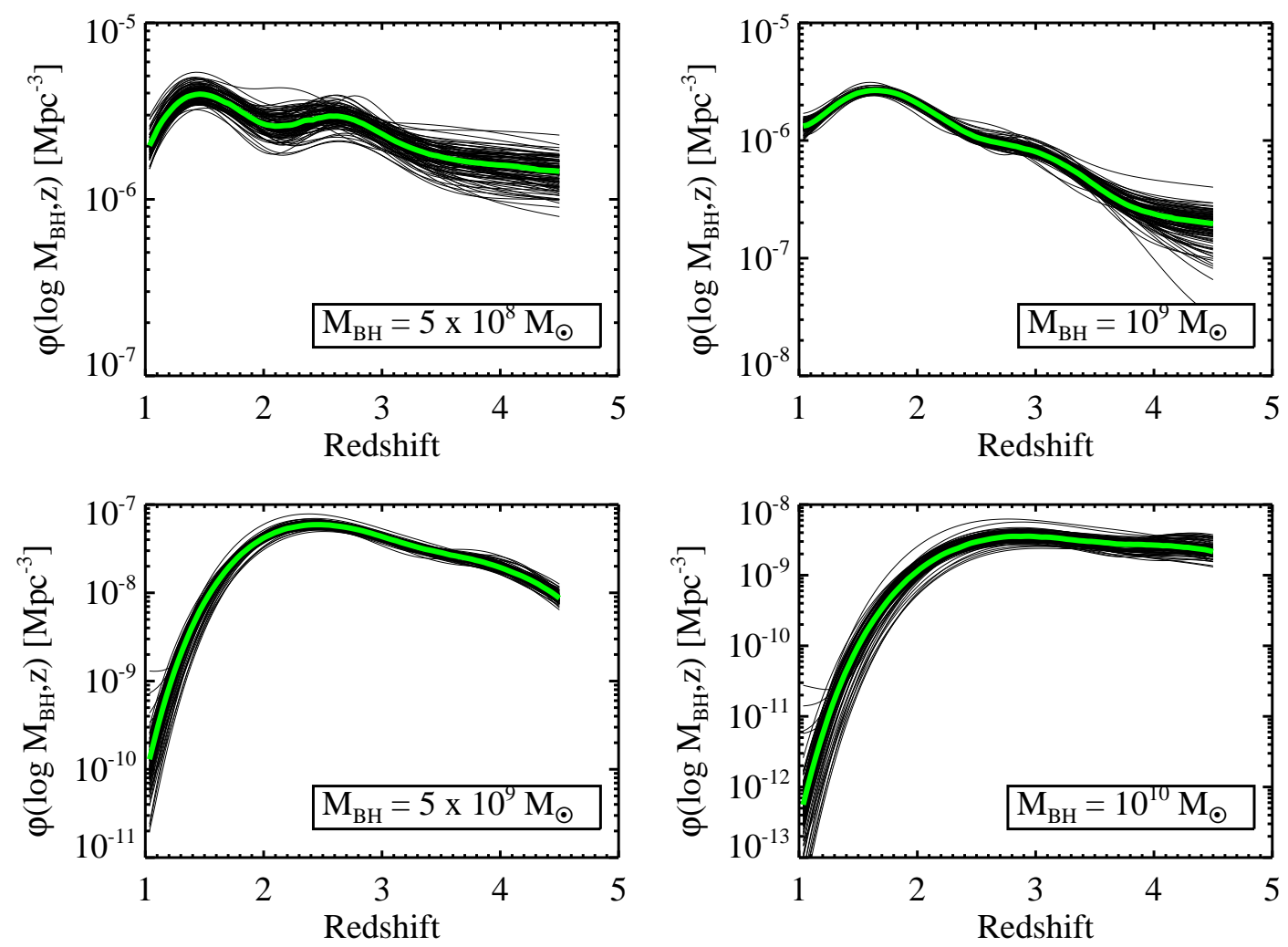

FIG. 5.- Evolution in the number density of BLQSO SMBHs for four different values of $M_{B H}$. A larger fraction of the most massive SMBHs are seen as BLQSOs at higher redshift, an effect commonly referred to as black hole downsizing. Downsizing of BLQSO SMBHs has also been seen in the LBQS (Vestergaard \& Osmer 2009). Symbols are as in Figure 3 The wiggles seen in the lowest mass bin are an artifact of fitting a Gaussian mixture model, unlikely to be real, and probably due to small unaccounted for errors in the selection function, which can become magnified due to the the fact that we are incomplete in this mass bin.

for incompleteness in flux, but not black hole mass. As a result, the $1 / V_{a}$ corrections only partially correct for incompleteness in $M_{B H}$, and the estimated binned BHMF will still suffer from incompleteness, especially at the low mass end. The two effects combined result in a systematic shift in the estimated BHMF toward higher $M_{B H}$ (Shen et al. 2008, KVF09). However, the Bayesian approach outlined in KVF09 is able to self-consistently correct for these two effects in a statistically rigorous manner, conditional on the survey selection function and assumptions implicit within the statistical model. In spite of these differences in methodology, our estimated BHMF agrees fairly well with that of Vestergaard et al. (2008) at the high mass end, considering the differences in the methodology, and the two do not strongly diverge until values of $M_{B H}$ where the SDSS is highly incomplete. The poorer agreement between the two estimates at $z<2$ is due to the fact that the BHMF is estimated using the $\mathrm{Mg}$ II emission line in this redshift range, which we find to have a higher statistical error than that of C IV. As a result, the our correction to the BHMF due to the error in the mass estimates is greater at these redshifts.

In Figure 5 we show the evolution in the comoving number density of SMBHs in BLQSO at four different masses. As is evident, the number density of higher mass BLQSO SMBHs peaks at higher redshift, where the number density of BLQSO SMBHs of $M_{B H} \sim 5 \times 10^{8} M_{\odot}$ peaks at $z \sim 1.5$, and the number density for $M_{B H} \sim 5 \times 10^{9} M_{\odot}$ peaks at $z \sim 2.5$. This result is consistent with what has commonly been referred to in the literature as black hole 'downsizing', where the most massive SMBHs are active, and grow, at earlier epochs than lower mass black holes, an effect also seen by Vestergaard \& Osmer (2009) and Steinhardt \& Elvis (2010a).

\subsection{The Broad Line Quasar Black Hole Mass Density, and Constraints on their Duty Cycle and Average Lifetime}

In Figure 6 we show the comoving mass density of SMBHs that reside in BLQSOs, as a function of redshift, $\rho_{Q S O}(z)$. The peak in the cosmic mass density of BLQSO SMBHs occurs at $z \sim 2$. Our constraint on the location of the peak in $\rho_{Q S O}(z)$ is consistent with the peak in the mass density derived from the Large Bright Quasar Survey and high- $z$ sample of Fan et al. (2001a), as calculated by Vestergaard \& Osmer (2009). Previous work has not found any evidence for evolution in the Eddington ratio distribution at $z>1$ for the most massive BLQSOs (e.g., McLure \& Dunlop 2004; Vestergaard 2004; Kollmeier et al. 2006; Vestergaard \& Osmer 2009). If there is no significant evolution in the Eddington ratio distribution at $z>1$ for these systems, then we would expect the peak in their luminosity density to coincide with the peak in their black hole mass density. The luminosity density of quasars peaks at $z \sim 2$ (e.g., Wolf et al. 2003; Hopkins et al. 2007b), matching the peak in black hole mass density observed in our work.

We can use the quasar BHMF to place constraints on the fraction of black holes that are seen in the BLQSO phase 


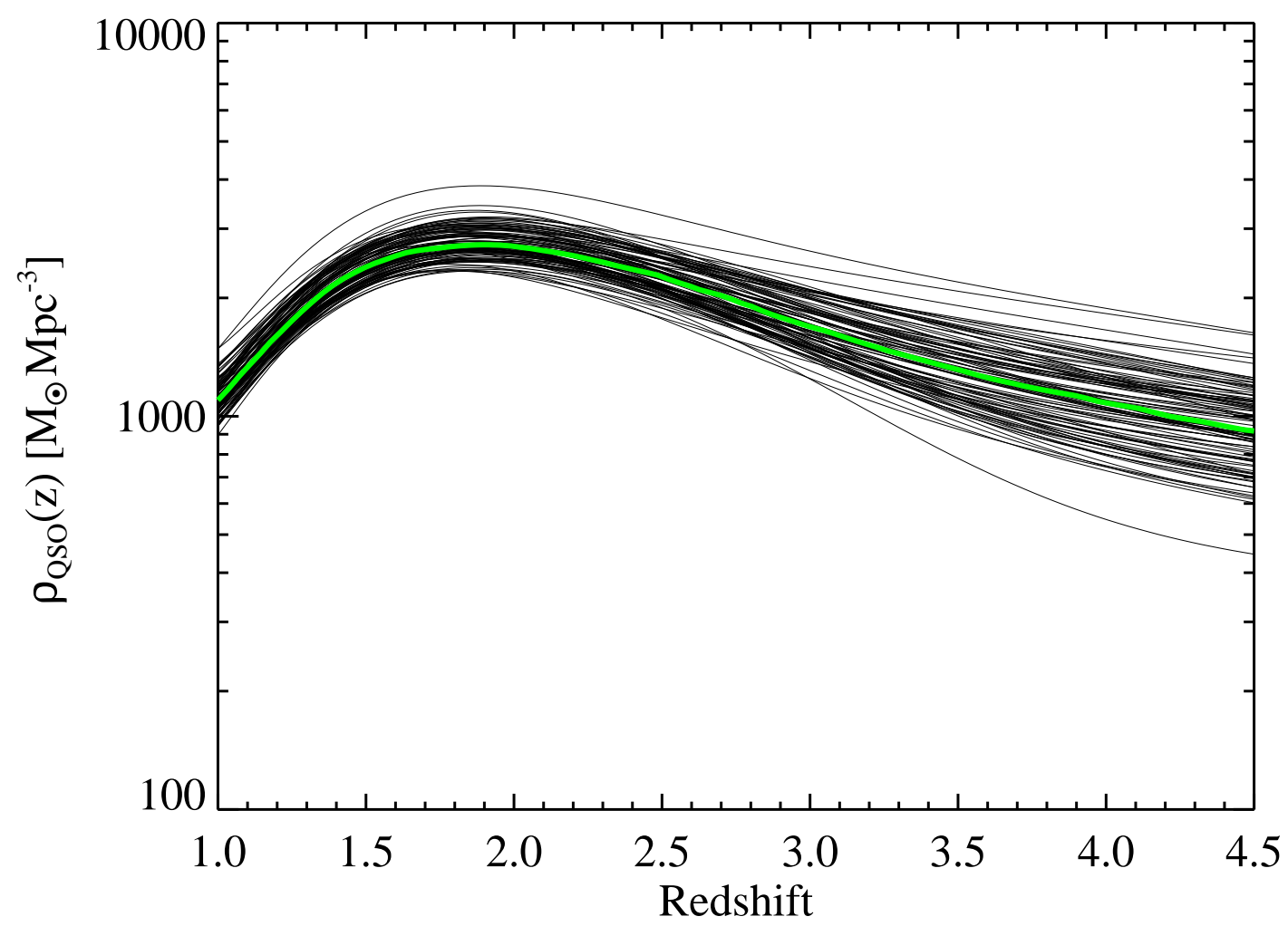

FIG. 6.- Evolution in the mass density of SMBHs seen as BLQSOs, symbols as in Figure 3 For comparison, recent estimates of the local mass density of all SMBHs suggest $\rho_{B H}(z=0) \sim 4 \times 10^{5} M_{\odot} \mathrm{Mpc}^{-3}$ (e.g. Yu \& Lu 2008). The mass density of SMBHs in BLQSOs peaks at $z \sim 2$.

as a function of $M_{B H}$; this quantity is commonly referred to as the BLQSO 'duty cycle'. Denote the duty cycle as $\delta\left(M_{B H}, z\right)$. Then

$$
\delta\left(M_{B H}, z\right) \equiv \frac{\phi_{Q S O}\left(M_{B H}, z\right)}{\phi_{B H}\left(M_{B H}, z\right)} .
$$

Here, $\phi_{B H}\left(M_{B H}, z\right)$ is the BHMF of all SMBHs at a given redshift. Ignoring mergers of SMBHs, we can compute a lower-limit to the duty cycle by comparing the BHMF at a certain redshift with the local SMBH number density, since $\phi_{B H}\left(M_{B H}, z\right) \leq \phi_{B H}\left(M_{B H}, 0\right)$. In Figure 7 we compute the lower limit of the BLQSO duty cycle at $z=1$ for SMBHs with $M_{B H}>5 \times 10^{8} M_{\odot}$. The duty cycle at $z=1$ is constrained to be $\delta \gtrsim 0.01$ at $M_{B H} \sim 10^{9} M_{\odot}$, falling steeply to $\delta \gtrsim 10^{-5}$ at $M_{B H} \sim 10^{10} M_{\odot}$. The decrease in the duty cycle with increasing black hole mass may be another reflection of SMBH downsizing, with the most massive BLQSO SMBHs being active at earlier cosmic epochs. Alternatively, it may be due to the fact that the most massive SMBHs spend a shorter amount of time in the broad line phase, as expected from some simulations of black hole feedback (e.g., Hopkins et al. 2006a). However, we note that the local number density of the most massive SMBHs is poorly constrained and subject to considerable systematic uncertainty (Lauer et al. 2007), and therefor duty cycle of BLQSOs with $M_{B H} \sim 10^{10} M_{\odot}$ may be subject to considerable systematic error. Indeed, the observed fall-off in the lower limit on the duty cycle for the most massive systems may simply be due to the fact that we are overestimating the number density of local SMBHs.

The quasar lifetime is not well constrained empirically (e.g., Martini 2004), but we can use our estimated BLQSO BHMF to estimate the BLQSO lifetime. For simplicity, we assume that all BLQSOs of a given mass have a single lifetime, $t_{B L}$. Furthermore, if a SMBH undergoes multiple episodes of BLQSO activity, then we assume that $t_{B L}$ is the same for each episode. These assumptions are unlikely to be true, but for the purposes of our work we may still think of $t_{B L}$ as a 'typical' BLQSO lifetime. Because the duty cycle is the probability of observing a SMBH as a BLQSO at a given $M_{B H}$ and redshift we can relate $t_{B L}$ to $\delta\left(M_{B H}, z\right)$. The probability of observing a SMBH as a BLQSO at redshift $z$ is the product of the probability that a SMBH becomes a BLQSO along our line of sight at some point in its evolution with the probability that that SMBH is a BLQSO between cosmic ages $t(z)-t_{B L}$ and $t(z)$, normalized by the probability that that SMBH is a BLQSO at $t \leq t(z)$. The normalization results from the additional assumption that if a SMBH of mass $M_{B H}$ exists at $t(z)$, and if it goes through at least one BLQSO episode as some point in its growth, then it had to have gone through at least one BLQSO episode at $t<t(z)$ in order to grow to $M_{B H}$ by $t(z)$. This is a reasonable assumption, at least for the most massive system, since all SMBHs at $t(z)$ had to grow from much lower mass seeds, and if BLQSO activity occurs for a SMBH at some point in its life, BLQSO activity would 


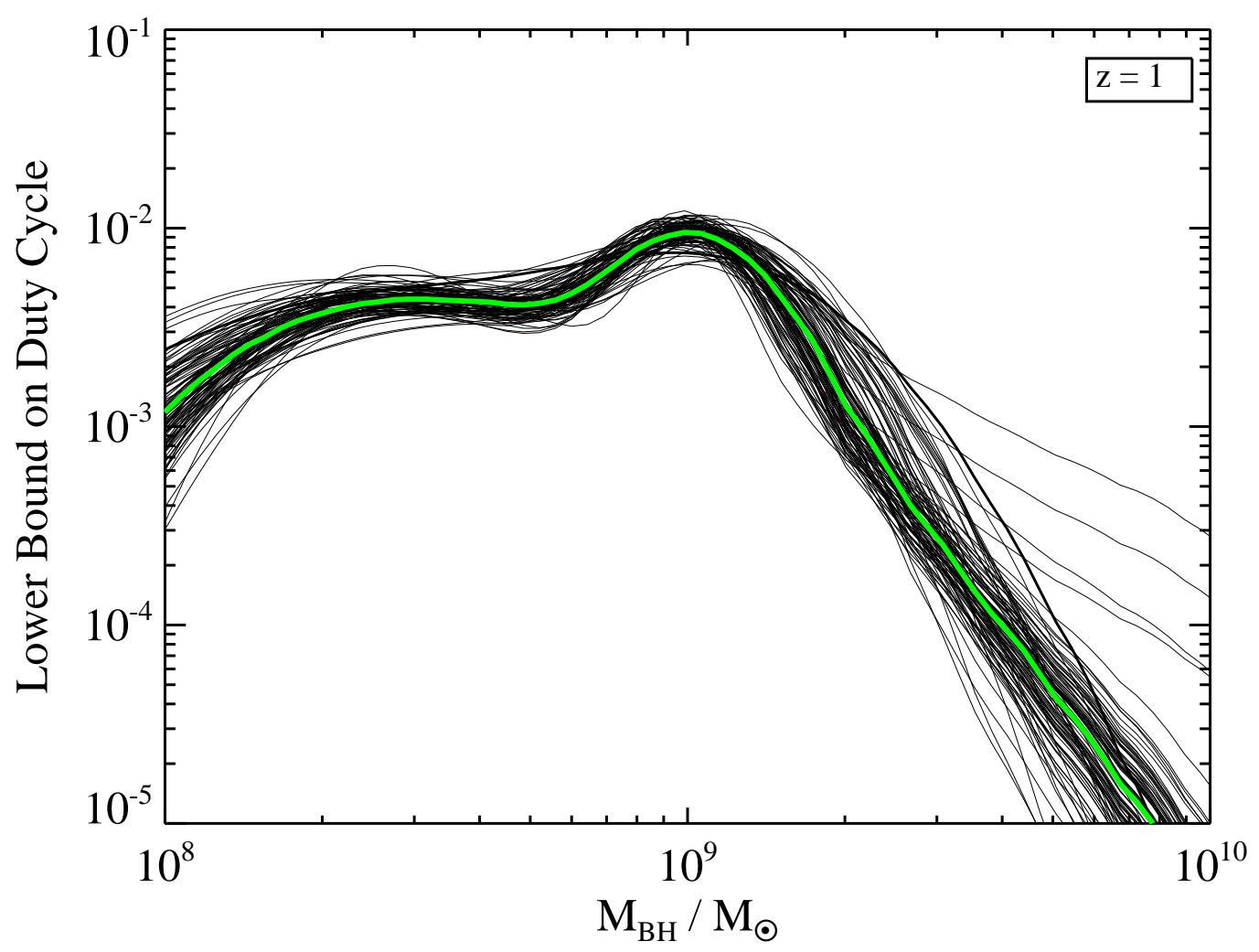

FIG. 7. - Lower bound on the duty cycle for BLQSO activity at $z=1$ as a function of $M_{B H}$, symbols as in Figure 3 The duty cycle for $M_{B H} \sim 10^{9} M_{\odot}$ BLQSO SMBHs at $z \sim 1$ is $\delta \gtrsim 0.01$, falling to $\delta \gtrsim 10^{-5}$ for $M_{B H} \sim 10^{10} M_{\odot}$. The decrease in the duty cycle with increasing black hole mass is likely due to either cosmic downsizing or a shorter BLQSO phase for the most massive SMBHs.

have occurred during this growth period. In other words, the assumption implicit in the normalization correction is that SMBH seeds of mass, say, $M_{B H} \sim 10^{9} M_{\odot}$, do not simply emerge and then undergo BLQSO activity at a cosmic epoch later than $t(z)$. The practical result of this assumption is that the BLQSO duty cycle of, say, SMBHs with $M_{B H} \sim 10^{9} M_{\odot}$, can decrease from $\delta \sim 1$ at $z \sim 7$ to $\delta \sim 10^{-3}$ at $z \sim 1$.

Denote the time that a BLQSO phase is initiated in a SMBH as $\tau$. Then, the probability that a SMBH BLQSO is seen between $t(z)-t_{B L}$ and $t(z)$ is the same as the probability that the time that a BLQSO phase was initiated for that source occured at $t(z)-t_{B L}<\tau<t(z)$. Note that this allows for multiple BLQSO episodes, as multiple phases of BLQSO episodes simply alter the probability of observing a SMBH as a BLQSO at a certain redshift. The duty cycle is thus related to $t_{B L}$ as

$$
\delta\left(M_{B H}, z\right)=\operatorname{Pr}\left(B L Q S O \mid M_{B H}\right)\left[\frac{\int_{t(z)-t_{B L}}^{t(z)} p\left(\tau \mid M_{B H}, B L Q S O\right) d \tau}{\int_{0}^{t(z)} p\left(\tau \mid M_{B H}, B L Q S O\right) d \tau}\right]
$$

where, $\operatorname{Pr}\left(B L Q S O \mid M_{B H}\right)$ is the probability that a SMBH with a mass of $M_{B H}$ is a BLQSO at some point in its evolution, and $p\left(\tau \mid M_{B H}, B L Q S O\right)$ is the probability distribution of BLQSO episode initiation time for a given black hole mass. Note that $\operatorname{Pr}\left(B L Q S O \mid M_{B H}\right)$ is not the probability that an object is currently seen as a BLQSO, but is the probability that it appears as a BLQSO to an observer on Earth at some point in its life. As mentioned above, $p\left(\tau \mid M_{B H}, B L Q S O\right)$ is sufficiently general to include both multiple and single episodes of BLQSO activity, although the actual form of $p\left(\tau \mid M_{B H}, B L Q S O\right)$ will depend on the distribution of the number of BLQSO episodes a SMBH goes through. All values of $M_{B H}$ in Equation (13) refer to the mass of the SMBH at redshift $z$.

If the distribution of $\tau$ does not evolve significantly over a time $t_{B L}$, then the integral in the numerator of Equation (13) can be approximated as $p\left(\tau \mid M_{B H}, B L Q S O\right) t_{B L}$. Likewise, if $p\left(\tau \mid M_{B H}, B L Q S O\right)$ is approximately constant over $t_{B L}$, then the distribution of BLQSO initiation times will be similar to the distribution of BLQSOs at the time we observed them. This is a reasonable assumption so long as $t_{B L}$ is short compared to the timescale for a significant change in the process that initiates BLQSO activity (e.g., mergers and other fueling events). Making this assumption, and rearranging Equation (13), it follows that

$$
t_{B L} \approx \frac{\delta\left(M_{B H}, z\right) \int_{0}^{t(z)} p\left(t^{\prime} \mid M_{B H}, B L Q S O\right) d t^{\prime}}{p\left(t(z) \mid M_{B H}, B L Q S O\right) \operatorname{Pr}\left(B L Q S O \mid M_{B H}\right)}
$$




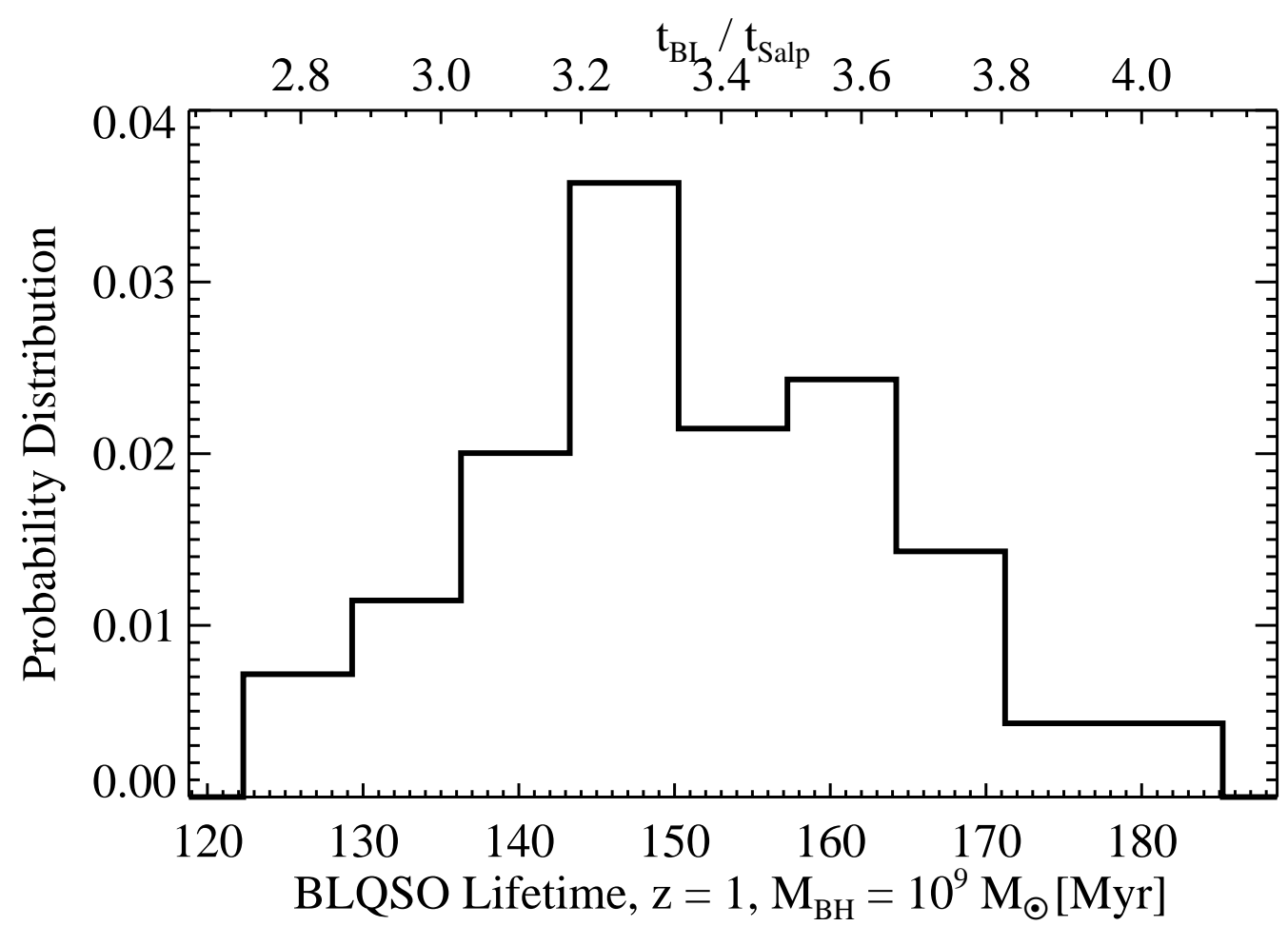

FIG. 8.- Probability distribution, given the observed data and assumptions of our analysis, of the lower bound on the BLQSO lifetime (Eq. 14) calculated at $z=1$ for $M_{B H}=10^{9} M_{\odot}$. The upper axis gives the lifetime in terms of the Salpeter timescale, which is $t_{S a l p}=$ $4.3 \times 10^{7} \mathrm{yrs}$ for a radiative efficiency of $\epsilon_{r}=0.1$. The lower-bound on the BLQSO lifetime is equal to the BLQSO lifetime if (1) all SMBHs of $M_{B H}=10^{9} M_{\odot}$ go through a BLQSO phase and (2) if the number density of these SMBHs at $z=1$ is not significantly less than the local number density. There is evidence that the latter condition is true (e.g., Merloni \& Heinz 2008), while the former condition is expected if the SMBH's growth is self-regulated (Hopkins \& Hernquist 2006).

where $p\left(t(z) \mid M_{B H}, B L Q S O\right)$ is the probability distribution of BLQSOs as a function of cosmic age, $t(z)$. The term $p\left(t \mid M_{B H}, B L Q S O\right)$ is related to the BHMF for BLQSOs according to

$$
p\left(t(z) \mid M_{B H}, B L Q S O\right)=\left|\frac{d t}{d z}\right|^{-1}\left[\frac{\phi\left(M_{B H}, z\right)}{\int_{0}^{\infty} \phi\left(M_{B H}, z\right) d M_{B H}}\right] .
$$

From Equation (14) it follows that for the special case where all SMBHs experience a BLQSO phase, and where BLQSOs initiation times are uniformly distributed over the age of the Universe, then $t_{B L}=\delta\left(M_{B H}, z\right) t(z)$. This is the definition of a quasar 'lifetime' commonly found in the literature; however, as the assumption of a uniform distribution of BLQSO initiation times is inconsistent with the BLQSO BHMF or luminosity function, $t_{B L}$ will in general not equal $\delta\left(M_{B H}, z\right) t(z)$ (see also Hopkins \& Hernquist 2009).

Because we have assumed that $t_{B L}$ is the same for all BLQSOs of a given mass, and therefore must be the same at all redshifts, Equation (14) may be calculated at any redshift. However, in reality we can only estimate a lower limit to $t_{B L}$. This is because we do not know the fraction of SMBHs that will experience a BLQSO phase, although if the SMBHs growth is self-regulated we might expect $\operatorname{Pr}\left(B L Q S O \mid M_{B H}\right) \approx 1$. However, if some SMBHs of mass $M_{B H}$ can never be seen as BLQSOs, possibly due to orientation-dependent obscuration, then $\operatorname{Pr}\left(B L Q S O \mid M_{B H}\right)<1$. In addition, as discussed above, we can only calculate a lower limit to $\delta\left(M_{B H}, z\right)$ by comparing the BLQSO BHMF at redshift $z$ with the local BHMF of all SMBHs. Moreover, implicit in these calculations is the assumption that none of these quantities depend strongly enough on mass to vary significantly during the growth that occurs in the BLQSO phase. In Figure 8 we show the probability distribution of the BLQSO age of SMBHs with $M_{B H}=10^{9} M_{\odot}$, calculated from Equation (14) at $z=1$. We calculate $t_{B L}$ at $M_{B H}=10^{9} M_{\odot}$ because our sample is reasonably complete at this mass, especially at this redshift. In addition, we chose $z=1$ because the local BHMF better approximates the $z=1$ BHMF than the BHMF at $z>1$, therefore giving the tightest lower bound on the duty cycle at $z=1$. We estimate $t_{B L}=150 \pm 15 \mathrm{Myr}$ as a lower bound on the age of the BLQSO phase for SMBHs of $M_{B H}=10^{9} M_{\odot}$. This estimate is roughly consistent with other estimates of quasar lifetimes (e.g., Yu \& Tremaine 2002; Martini 2004; Goncalves et al. 2008; Hopkins \& Hernquist 2009). 

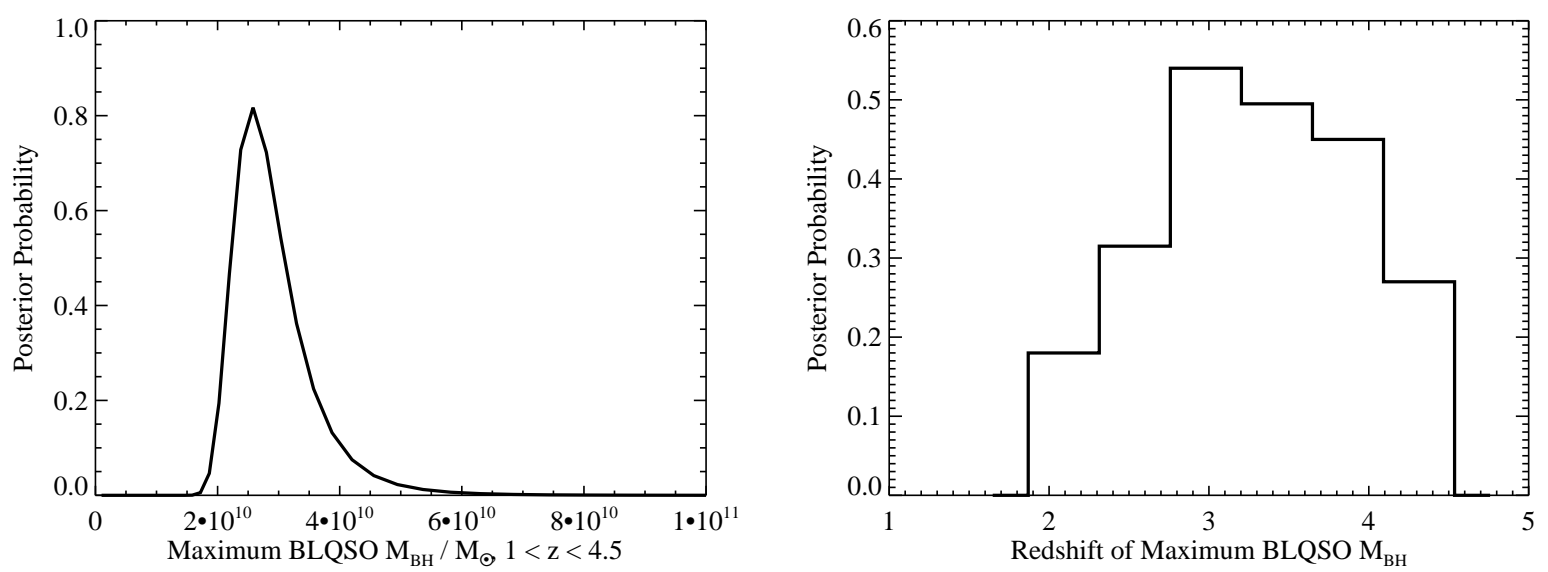

FIG. 9.- Probability distribution of $M_{B H}$ for the most massive SMBH that could be observed as a BLQSO at $1<z<4.5$ (left), and the probability distribution for the redshift that this SMBH would be seen as a BLQSO (right). Here, we do not interpret $M_{B H}^{M a x}$ to represent a hard physical upper limit to $M_{B H}$, but rather it is the result of a finite number of black holes drawn from a mass function. The constraints on $M_{B H}^{M a x}$ that we have obtained are consistent with previous observational work, but this is the first time that rigorous constraints on $M_{B H}^{M a x}$ and its redshift have been obtained. The most likely value for $M_{B H}^{M a x}$ is $M_{B H} \approx 2.5 \times 10^{10} M_{\odot}$, and this SMBH would likely be seen as a BLQSO at $z \gtrsim 2$.

The probability distribution for the maximum mass of a SMBH in a BLQSO may place important constraints on models of SMBH growth, and may be calculated directly from the BHMF. In the context of our work, we do not consider the maximum mass of a SMBH to be caused by a hard upper limit, above which it is impossible to make a more massive black hole, but rather the result of a finite number of black holes drawn from a mass function. The probability that the maximum mass of a SMBH in a sample of $N$ BLQSOs is less than $\mathcal{M}$ is simply given by the probability that all $N$ SMBHs have $M_{B H}<\mathcal{M}$ :

$$
\operatorname{Pr}\left(M_{B H}^{M a x}<\mathcal{M} \mid N\right)=\left[\operatorname{Pr}\left(M_{B H}<\mathcal{M}\right)\right]^{N}
$$

Note that $N$ is the total number of BLQSOs that could be observed in an all-sky survey with no flux limit; i.e., $N$ is the normalization of the BLQSO BHMF. The term $\operatorname{Pr}\left(M_{B H}<\mathcal{M}\right)$ is calculated from the BHMF as

$$
\operatorname{Pr}\left(M_{B H}<\mathcal{M}\right)=\frac{1}{N} \int_{0}^{\mathcal{M}} \int_{0}^{\infty} \phi\left(M_{B H}, z\right)\left(\frac{d V}{d z}\right) d z d M_{B H}
$$

The probability distribution of $M_{B H}^{M a x}$ is then found by differentiating Equation (16) with respect to $\mathcal{M}$ and evaluating the result at $\mathcal{M}=M_{B H}^{M a x}$ :

$$
p\left(M_{B H}^{M a x} \mid N\right)=\left[\operatorname{Pr}\left(M_{B H}<M_{B H}^{M a x}\right)\right]^{N-1} \int_{0}^{\infty} \phi\left(M_{B H}, z\right)\left(\frac{d V}{d z}\right) d z .
$$

The posterior probability distribution for $M_{B H}^{M a x}$, given the observed data, can be calculated by averaging Equation (18) over the MCMC output.

In Figure 9 we show the posterior probability distribution of the maximum SMBH in a BLQSO at $1<z<4.5$. Here, $M_{B H}^{M a x}$ should be interpreted as the maximum mass of a SMBH in a BLQSO that would be observed in an all-sky survey without a flux limit over the redshift interval $1<z<4.5$. Thus, $M_{B H}^{M a x}$ is a lower bound on the mass of the most massive SMBH in the Universe. In addition, in Figure 9 we also show the probability distribution of the redshift at which the quasar with $M_{B H}^{M a x}$ would be found. As can be seen from these figures, the maximum mass of a SMBH in a BLQSO at $1<z<4.5$ is $M_{B H}^{M a x} \sim 3 \times 10^{10} M_{\odot}$. The probability distribution for the redshift of $M_{B H}^{\operatorname{Max}}$ is rather broad, but we constrain the redshift for the BLQSO hosting this SMBH to be $z \gtrsim 2$.

Our results are in agreement with what others have found using samples of broad line mass estimates (e.g., Vestergaard 2004; Vestergaard et al. 2008; Netzer et al. 2007), although Labita et al. (2009a,b) find a smaller value of $M_{B H}^{M a x} \sim 5 \times 10^{9} M_{\odot}$. However, in the model of Labita et al. (2009a) $M_{B H}^{M a x}$ is a parameter determining the shape of the mass function, and is not the actual maximum black hole mass of a sample of objects drawn from the distribution of black hole mass; i.e., there is nothing in the statistical model of Labita et al. (2009a) that prevents objects with $M_{B H}>M_{B H}^{M a x}$. As such, the actual realized maximum mass in a large sample of objects will be greater than the value of $M_{B H}^{M a x}$ estimated using the model of Labita et al. (2009a), as we have found. Considering this, our results are consistent with those of Labita et al. (2009a, b).

These highly massive black holes represent the extremes of black hole growth, and thus are important for constraining models of black hole growth. Furthermore, these massive black holes offer the best chance of probing the faint end of the BLQSO Eddington ratio distribution. Therefore, it is of use to investigate how large and deep a survey must be 


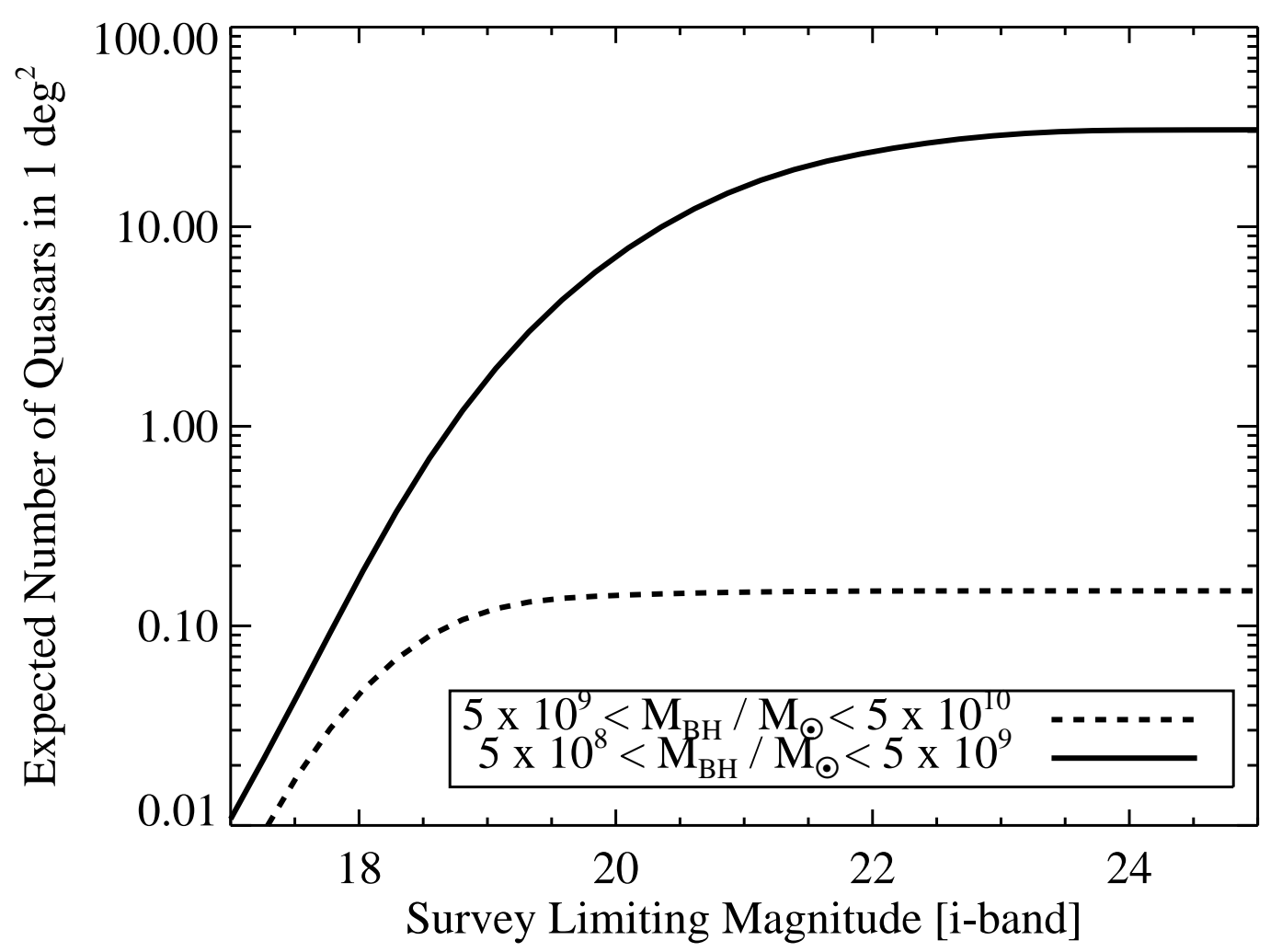

Fig. 10. - Expected number counts of BLQSOs at $1<z<4.5$ with black hole masses $5 \times 10^{8}<M_{B H} / M_{\odot}<5 \times 10^{9}$ (solid line) and $5 \times 10^{9}<M_{B H} / M_{\odot}<5 \times 10^{10}$ (dashed line) in a $1 \mathrm{deg}^{2}$ survey as a function of limiting magnitude. One would need to search an angular area $\Omega>10 \mathrm{deg}^{2}$ in order to expect to find at least one BLQSO with $M_{B H} \sim 10^{10} M_{\odot}$. In addition, one does not become complete at $M_{B H} \sim 5 \times 10^{8} M_{\odot}$ until $i \sim 23$.

in order to find an useful number of these objects. In Figure 10 we show the expected number of high mass SMBHs in BLQSOs at $1<z<4.5$ in a $1 \mathrm{deg}^{2}$ survey as a function of limiting $i$-magnitude, assuming our best-fit statistical model. We stress that these are the expected number counts for the true black hole masses, and not the mass estimates. Because the errors in the mass estimates will scatter more sources into higher mass bins than into lower mass bins, the number of sources with estimated mass in a mass bin will be larger than the actual number of sources.

Any survey with a flux limit of $i<19$ should be able to detect BLQSOs with $M_{B H} \gtrsim 5 \times 10^{9} M_{\odot}$; however, the survey must have an area of $\Omega \gtrsim 10 \mathrm{deg}^{2}$ to expect to detect at least one of these objects. Similarly, in order to get a large number of BLQSOs with $M_{B H} \gtrsim 5 \times 10^{8} M_{\odot}$, one needs a deep, large area spectroscopic survey. For example, the BLQSO spectroscopic samples from the COSMOS survey (Scoville et al. 2007) cover $\approx 2 \operatorname{deg}^{2}$ at $i<24$ (Trump et al. 2007) and $i<22.5$ for zCOSMOS (Merloni et al. 2010), and, ignoring redshift incompleteness, are therefore expected to contain $\sim 60$ BLQSOs at $1<z<4.5$ with masses $5 \times 10^{8}<M_{B H} / M_{\odot}<5 \times 10^{9}$, but none with masses $M_{B H}>5 \times 10^{9} M_{\odot}$.

\subsection{Implied Eddington Ratio Distributions}

As discussed in $\S 3.1$, we also estimate the distribution of the ratio of the Eddington ratio to the bolometric correction at $1350 \AA, \Gamma_{E d d} / C_{1350}$. Under the assumption that $\Gamma_{E d d} / C_{1350}$ follows a mixture of log-normal distributions, with mean values that depends linearly on $\log M_{B H}$, and that $C_{1350}=4.3$ for all sources (Vestergaard \& Osmer 2009), our model implies that the geometric mean Eddington ratio increases with $M_{B H}$ according to:

$$
\left\langle\frac{L}{L_{E d d}}\right\rangle_{\text {geo }}=0.12 \pm 0.01\left(\frac{M_{B H}}{M_{\odot}}\right)^{0.48 \pm 0.04}
$$

The dispersion in $L / L_{E d d}$ decreases with increasing $M_{B H}$, having a value of $\sim 0.4$ dex at $M_{B H} \sim 10^{8} M_{\odot}$, and decreasing to $\sim 0.3$ dex at $M_{B H} \sim 10^{9} M_{\odot}$. Both the dispersion and slope are smaller than what was found by KVF09, who analyzed a sample of BLQSOs from the Bright Quasar Survey at $z<0.5$, suggesting possible evolution in the Eddington ratio distribution. However, the statistical significance of a difference in the slopes is marginal at best, as the differences are only significant at $\sim 2 \sigma$.

In Figure 11 we show the implied distribution of BLQSO Eddington ratios assuming a constant bolometric correction 


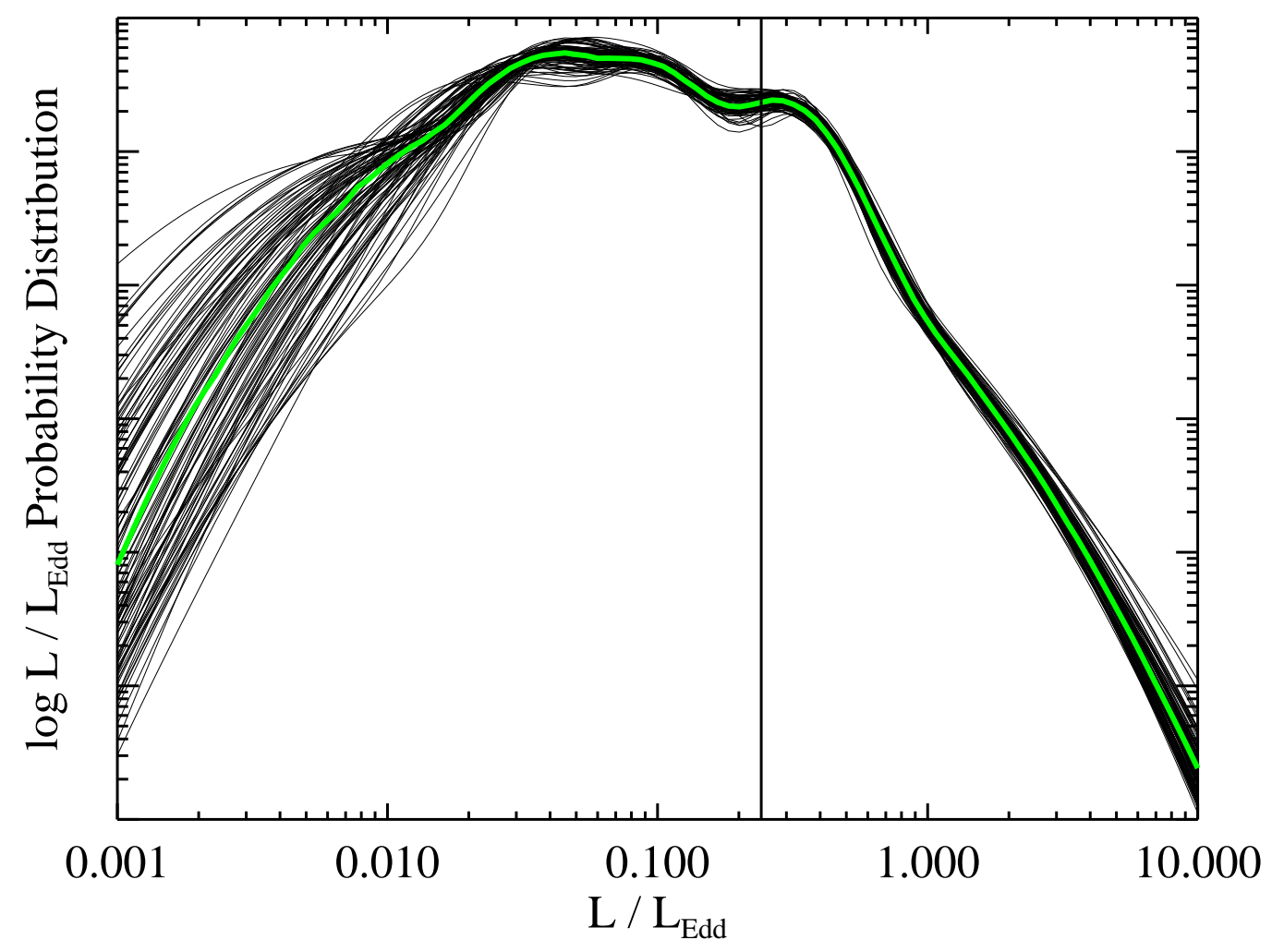

FIG. 11. - Inferred Eddington ratio distribution, assuming the mixture of log-normals form of Equation (2) and a bolometric correction of $C_{1350}=4.3$. The vertical line marks the $10 \%$ completeness limit for the SDSS DR3 sample at $z=1$; Figure 4 shows that the $10 \%$ completeness limits are similar for $z \sim 1,3$, and 4 , but shallower for $z \sim 2$. Our inferred Eddington ratio distribution peaks near $L / L_{E d d} \sim 0.05$ and has dispersion of $\sim 0.4$ dex, although the peak fall below the $10 \%$ completeness limit and should be interpreted with caution. Our inferred Eddington ratio distribution is shifted toward lower values of $L / L_{E d d}$ and has a higher dispersion than previous work, which did not correct for incompleteness. Our estimated Eddington ratio distribution suggests that most SMBHs in BLQSOs are not radiating near their Eddington limit.

of $C_{1350}=4.3$ (Vestergaard \& Osmer 2009). As can be seen, the estimated distribution of quasar Eddington ratios peaks at $L / L_{E d d} \sim 0.05$. However we note that the location of the Eddington ratio peak falls below the $10 \%$ completeness limit of the SDSS, and thus the exact location of the peak is highly uncertain. Our inferred Eddington ratio distribution is broader and shifted towards smaller values of $L / L_{E d d}$ than what has been found in previous work, which were not able to fully correct for incompleteness in black hole mass and Eddington ratio (e.g., McLure \& Dunlop 2004; Vestergaard 2004; Kollmeier et al. 2006). However, it is consistent with what Shen et al. (2008) found using a similar approach to ours. The major differences between our work and that of Shen et al. (2008) is that they assume a power-law distribution of $M_{B H}$, and they constrain the BHMF and Eddington ratio distribution by visually matching the model distributions to the observed distributions. Our results, as well as the results of (Shen et al. (2008), show that the narrow distribution in quasar Eddington ratios seen in previous work, and peaking at $L / L_{E d d} \sim 0.25$, is due to uncorrected incompleteness. Indeed, deeper samples of BLQSOs have observed Eddington ratio distributions that are broader and peak at lower values (Gavignaud et al. 2008; Trump et al. 2009). Therefore, we conclude that most BLQSOs are not radiating at or near the Eddington limit, and that there is a large dispersion in Eddington ratio for BLQSOs.

Recently, Steinhardt \& Elvis (2010a) have analyzed the SDSS DR5 sample of mass estimates calculated by Shen et al. (2008), and concluded that there is a dirth of objects at high Eddington ratio, and that there is a redshift-dependent systematic decrease in Eddington ratio with increasing black hole mass for BLQSOs in the high $L / L_{E d d}$ tail of the distribution. They have called this effect a sub-Eddington boundary. While we also find evidence that BLQSOs radiating near the Eddington limit are rare, as did KVF09, the dependence on black hole mass is seemingly in contrast to what we find here, in that we find that the average Eddington ratio increases with $M_{B H}$, assuming a constant bolometeric correction. The most important difference between their work and ours is the fact that Steinhardt \& Elvis (2010a) analyze seperate redshift bins, while we do not model a redshift dependence in the Eddington ratio distribution. Steinhardt \& Elvis (2010a) did not perform any calculations for the whole sample, averaged over all redshifts, making a more direct comparision difficult, and thus it is unclear how discrepant our conclusions are.

Another potential contributor to this discrepancy is the handling of the statistical error in the mass estimates. Statistical errors (e.g., measurement error) have the effect of flattening slopes and correlations when one analyzes the quantity contaminated by the error (e.g., Akritas \& Bershady 1996; Kelly 2007; Kelly \& Bechtold 2007), due to the 
fact that the distribution of the estimated quantity is a biased estimate of the distribution of the quantity of interest. Because the mass estimates are contaminated by statistical error, the dependence of luminosity on the mass estimates will be flatter than the intrinsic dependence of luminosity on $M_{B H}$. In particular, the statistical error will cause some masses to be underestimated, and some to be overestimated. The objects which appear to have the highest masses at a given luminosity will have the highest overestimates, and thus will have their Eddington ratios underestimated. As a result, there will be an apparent dirth of high Eddington ratio objects at the highest masses. Similarly, there will be an apparent excess of high Eddington ratio objects in the low mass bins. Our method estimates the intrinsic dependence of luminosity on $M_{B H}$ by, in a sense, 'deconvolving' the distribution of the mass estimates and luminosity with the distribution of the error in the mass estimates; this is also why we find a somewhat steeper decline in the mass function at the highest masses, as compared to the estimate reported by Vestergaard et al. (2008).

Although the statistical errors in the mass estimates can have the affect described above, possibly contributing to the different conclusions, it is unlikely that the statistical errors alone are sufficient to account for the discrepancy, and other possibilities include differences in the methods and scaling relationship employed for estimating the masses, and other differences in the methods of data analysis employed. However, further investigation is beyond the scope of our work. In addition, we note that our samples only overlap at $1<z<2$, and we cannot compare with the low redshift results of Steinhardt \& Elvis (2010a), where they find the greatest evidence for these effects.

Although we have used a flexible form for $p\left(L \mid M_{B H}\right)$, our estimated distribution for $L / L_{E d d}$ may be affected by significant systematics, as it relies on the assumption of a constant bolometric correction. There is evidence that the bolometric correction depends on both Eddington ratio (Vasudevan \& Fabian 2007; Young et al. 2010) and black hole mass (Kelly et al. 2008), and the increase of $L / L_{E d d}$ with $M_{B H}$ we find may instead be a reflection of a decrease in the bolometeric correction with $M_{B H}$. Indeed, Kelly et al. (2008) find that the ratio of optical/UV flux to X-ray flux increases with $M_{B H}$, implying that the bolometeric correction to the UV flux decreases with $M_{B H}$, and therefore we would infer an increase in $L / L_{E d d}$ with $M_{B H}$ assuming a constant bolometeric correction, as we do. In addition to these issues, we also note that the SDSS is at least partially incomplete at all Eddington ratios at $z>1$ [1], as shown in Figure 4, and further work is needed using deeper surveys to confirm these results.

\section{DISCUSSION}

\subsection{Connection with Quasar Lightcurves}

A significant amount of recent work has suggested that quasar feedback regulates the growth of SMBHs and affects the large-scale evolution of its host galaxy. Understanding the quasar lightcurve is thus of fundamental importance for understanding black hole growth and feedback, as well as placing constraints on the physics of quasar accretion flows. The distribution of luminosity at a given black hole mass can be related to the quasar lightcurve, and therefore one can use the estimated distribution of luminosity at a given black hole mass to place some empirical constraints on models for quasar lightcurves. We address this in the following section, and argue that our estimated Eddington ratio distribution is consistent with models where the BLQSO phase represents the final stages of a SMBH's growth, in which the QSO lightcurve is expected to decay until the object no longer appears as a BLQSO.

For a given fueling episode, denote the quasar lightcurve as $L(t)$, and the mass fueling rate to the SMBH as a function of time as $\dot{M}(t)$. The fueling rate, $\dot{M}(t)$, gives the rate at which matter is externally supplied to the BLQSO accretion disk. The probability distribution of quasar luminosity at a given $M_{B H}, \dot{M}$, and $t$ is $p\left(L \mid M_{B H}, \dot{M}, t\right)$, and the probability distribution of the fueling rate at a given $M_{B H}$ and $t$ is $p\left(\dot{M} \mid M_{B H}, t\right)$. The distribution $p\left(L \mid M_{B H}, \dot{M}, t\right)$ is determined by the physics of the accretion disk, since the quasar luminosity is produced by viscous stresses in the accretion disk. For example, Siemiginowska \& Elvis (1997) calculate $p\left(L \mid M_{B H}, \dot{M}, t\right)$ implied by the model of Siemiginowska et al. (1996) for a thermal-viscous accretion disk stability. The distribution $p\left(\dot{M} \mid M_{B H}, t\right)$, on the other hand, depends on the physics and stochastic nature of the fueling mechanism and quasar feedback.

Because the quasar luminosity is determined by the physics of the accretion disk, it is reasonable to assume that given $M_{B H}$ and $\dot{M}, L$ is independent of time. This does not imply that the quasar lightcurve does not vary with time, as it certainly does, but rather that knowing the value of $t$ does not give us any additional information on the value of $L$ when we already know $M_{B H}$ and $\dot{M}$ at a given $t$. We therefore drop the explicit dependence of $p\left(L \mid M_{B H}, \dot{M}, t\right)$ on time.

The distribution of luminosity at a given $M_{B H}$ is calculated as

$$
p\left(L \mid M_{B H}\right)=\int_{0}^{\infty} p\left(L \mid \dot{M}, M_{B H}\right)\left[\int_{t_{0}}^{t_{o}+t_{B L}} p\left(\dot{M} \mid t, M_{B H}\right) p\left(t \mid M_{B H}\right) d t\right] d \dot{M},
$$

where $p\left(t \mid M_{B H}\right)$ is the probability of seeing a BLQSO at time $t$, given $M_{B H}$, and $t_{B L}$ is the length of time that a quasar would be classified as a BLQSO. Here we have defined the broad line phase of quasar activity to start at $t=t_{0}$. The term $p\left(t \mid M_{B H}\right)$ is related to the model for black hole growth, since $p\left(t \mid M_{B H}\right) \propto p\left(M_{B H} \mid t\right) p(t)$. Because we observe quasars randomly during their BLQSO phase, $p(t)$ is uniform and $p\left(t \mid M_{B H}\right) \propto p\left(M_{B H} \mid t\right)$. In general, BLQSOs with larger values of $M_{B H}$ are more likely to be seen later in their lifetime, i.e., at larger values of $t$.

\footnotetext{
${ }^{10}$ Steinhardt \& Elvis (2010a) also included quasars at $z>2$ in some of their plots, but their calculations and conclusions were based on quasars at $z<2$ because of concerns regarding the validity of using C IV in the mass estimates.

${ }^{11}$ As discusses earlier, this is merely a reflection of the fact that the mass function increases steeply toward lower masses, which are hard to detect even if these low mass SMBHs are radiating near the Eddington limit
} 
As an alternative to Equation (20), $p\left(L \mid M_{B H}\right)$ may be directly calculated from the amount of time that a BLQSO spends above a given luminosity, as a function of $M_{B H}$. In this case, the term $p\left(L \mid M_{B H}\right)$ is directly related to the 'luminosity-dependent' lifetime interpretation advocated by Hopkins et al. (2005b. C), where the quasar 'lifetime' is the amount of time that a quasar spends above a given luminosity. Assume that $p\left(t \mid M_{B H}\right) \propto 1$ for BLQSOs, and denote $T\left(L \mid M_{B H}\right)$ to be the amount of time a BLQSO spends above a luminosity $L$, as a function of $M_{B H}$. Then

$$
p\left(L \mid M_{B H}\right) \propto\left|\frac{d T\left(L \mid M_{B H}\right)}{d L}\right| .
$$

For example, Hopkins \& Hernquist (2009) suggest that $p\left(L \mid M_{B H}\right)$ can be well approximated as a Schechter function, which generalizes the distributions implied by several common models of quasar lightcurves often found in the literature.

\subsubsection{Effects of Evolution in the Quasar Fueling Rate}

Recent work on quasar fueling and feedback suggests that the BLQSO phase occurs at the end of the fueling event, during which feedback energy from the AGN is able to unbind the surrounding gas, removing the obscuring material and halting the black hole's growth. Within the context of this model, the SMBH does not experience a large fractional increase in mass beyond the value of $M_{B H}$ that is observed during its time as a BLQSO. Therefore, we approximate $p\left(t \mid M_{B H}\right)$ as being uniform and independent of $M_{B H}$, i.e., $p\left(t \mid M_{B H}\right)=1 / t_{B L}$. In addition, this model predicts that during this so-called 'blow-out' phase, the fuel supply is set by the evolution of the blastwave caused by the injection of energy from the SMBH, and is expected to be of the form $\dot{M}(t) \propto t^{-\beta}$ (Hopkins \& Hernquist 2006; Hopkins et al. 2006a). Alternatively, if the fuel supply is suddenly removed, then the accretion rate during the broad line phase is due to evolution of the viscous accretion disk. The fueling rate also has a power-law form under viscous evolution of the disk, but with a different value for $\beta$ (Cannizzo et al. 1990; Pringle 1991). Under these models, it is reasonable to approximate the fuel supply as a deterministic process, $\dot{M}(t) \propto t^{-\beta}$. If the evolution of the fuel supply is a deterministic and one-to-one process, and assuming that $p\left(t \mid M_{B H}\right) \propto 1 / t_{B L}$, then $p\left(\dot{M} \mid M_{B H}\right)$ is

$$
p\left(\dot{M} \mid M_{B H}\right)=\frac{1}{t_{B L}} \frac{d t\left(\dot{M}, M_{B H}\right)}{d \dot{M}},
$$

where $t\left(\dot{M}, M_{B H}\right)$ is the time implied by $\dot{M}\left(t, M_{B H}\right)$, i.e., $t\left(\dot{M}, M_{B H}\right)$ is the inverse function of $\dot{M}\left(t, M_{B H}\right)$. If the quasar lightcurve is a many-to-one function, then the right side of Equation (22) is replaced by a sum of derivatives, as in Equation (15) of $\mathrm{Yu} \& \mathrm{Lu}$ (2004). Inserting Equation (22) into Equation (20) and dropping the time integral gives 12

$$
p\left(L \mid M_{B H}\right)=\frac{1}{t_{B L}} \int_{0}^{\infty} p\left(L \mid \dot{M}, M_{B H}\right) \frac{d t\left(\dot{M}, M_{B H}\right)}{d \dot{M}} d \dot{M} .
$$

For models where $\dot{M}(t) \propto t^{-\beta}$, a power-law distribution of fueling rates follows from Equation (22) $p\left(\dot{M} \mid M_{B H}\right) \propto$ $\dot{M}^{-(1+1 / \beta)}$ (see also Eq. 43 in Yu \& Lu 2008). Therefore, assuming $\dot{M}(t) \propto t^{-\beta}$ gives

$$
p\left(L \mid M_{B H}\right) \propto \int_{0}^{\infty} p\left(L \mid \dot{M}, M_{B H}\right) \dot{M}^{-(1+1 / \beta)} d \dot{M} .
$$

The feedback-driven model of Hopkins \& Hernquist (2006) predicts a value of $\beta \sim 2$, while the disk evolution model predicts $\beta \sim 1.2$ (Cannizzo et al. 1990; Yu et al. 2005; King \& Pringle 2007), implying that $p\left(\dot{M} \mid M_{B H}\right) \propto \dot{M}^{-1.5}$ or $p\left(\dot{M} \mid M_{B H}\right) \propto \dot{M}^{-1.67}$, respectively.

\subsubsection{Effects of Time-Dependent Accretion Disks}

It is apparent from Equation (24) that the effect of time-dependent accretion disk behavior is to create a distribution of luminosities at a given $M_{B H}$ that is flatter than that expected from a simple power-law decay in the fueling rate. While the BLQSO fueling rate may be approximated as a deterministic process, quasar lightcurves are observed to exhibit aperiodic and stochastic variations across all wavelengths (for a review see Ulrich et al. 1997). Therefore, the quasar lightcurve will be stochastic, i.e., the value of the luminosity is not completely determined by $M_{B H}$, and $\dot{M}$. From Equation (24) it can be observed that if we assume a deterministic relationship between $L$ and $\dot{M}$, i.e., a time-steady accretion disk, as much previous work on SMBH growth and feedback has assumed, then $L \propto \dot{M}$ and the quasar lightcurve simply traces the fueling rate evolution. In this case, $p\left(L \mid M_{B H}, \dot{M}\right)$ is a delta function and $p\left(L \mid M_{B H}\right) \propto L^{-(1+1 / \beta)}$. However, the time-dependent and stochastic nature of the accretion disk emission broadens the observed luminosity function beyond that implied solely from the BLQSO fueling evolution.

Although quasars are observed to vary at all wavelengths, we focus our remaining discussion on optical variability, since we are interested in the distribution of optical luminosities in this work. Kelly et al. (2009a) found that quasar optical lightcurves on timescales $\lesssim 7$ yrs in the rest frame of the quasar are well-described by a Gaussian process

\footnotetext{
12 This follows from $p\left(L \mid M_{B H}\right)=\int p\left(L \mid \dot{M}, M_{B H}\right) p\left(\dot{M} \mid M_{B H}\right) d \dot{M}$. Alternatively, we could have arrived at Eq. 23 by taking $p\left(\dot{M} \mid M_{B H}, t\right)$ to be a delta function centered at the value of $\dot{M}$ determined by $t$, and directly doing the integration in Eq. 20.
} 
on the logarithmic scale (see also Kozłowski et al. 2010), with characteristic timescale consistent with accretion disk thermal time scales. They suggested that quasar variability on these time scales is due to a turbulent magnetic field in the accretion disk, which drives changes in the radiation energy density of the disk, as also seen in 3-dimensional magneto-hydrodynamic simulations of radiation-pressure dominated accretion disks (Hirose et al. 2008). Extrapolation of their best-fit lightcurves imply that flux variations for this particular process have a standard deviation $\sim 0.1$ dex on timescales long compared to the disk thermal time scale, independent of $M_{B H}$. If this is the only source of quasar optical variability, then $p\left(L \mid M_{B H}, \dot{M}\right)$ is a gaussian distribution centered at $L_{o p t}=C_{o p t} \epsilon_{r} \dot{M} c^{2}$ with standard deviation $\sim 0.1$ dex. Here $C_{o p t}$ is the bolometric correction to the optical luminosity (which likely depends on $M_{B H}$ and $\left.\dot{M}\right), \epsilon_{r}$ is the radiative efficiency, and $c$ is the speed of light. This process only produces a small amount of broadening in the luminosity distribution, relative to that implied solely from the BLQSO fueling evolution. Therefore, $p\left(L \mid M_{B H}\right) \propto L^{-(1+1 / \beta)}$ is a reasonable approximation when the fuel rate declines as a power-law, so long as there are no other variability components in the accretion disk beyond that observed by Kelly et al. (2009a). However, this does not avoid the issue of a non-constant bolometric correction.

Variability on longer time scales may be driven by accretion disk instabilities. Accretion disks based on the standard $\alpha$-prescription for viscosity (Shakura \& Sunyaev 1973) are subject to a number of accretion disk instabilities that can potentially have a significant effect on the quasar lightcurve. At high accretion rates $(\dot{m} \gtrsim 0.025)$ a radiationpressure instability may operate on time scales $\gtrsim 10^{4}$ yrs (Czerny et al. 2009), while at lower accretion rates a thermal-viscous ionization instability (e.g., Lin \& Shields 1986; Siemiginowska et al. 1996; Menou \& Quataert 2001; Janiuk et al. 2004) may operate on time scales $\gtrsim 10^{6}$ yrs. The ionization instability has been invoked to explain the outbursts seen in dwarf novae and soft X-ray transients (for a review see Lasota 2001), and the radiation pressure instability has been invoked to explain the outbursts seen in the microquasar GRS 1915+105 (e.g., Nayakshin et al. 2000; Janiuk et al. 2000). Moreover, Goodman \& Tan (2004) suggest that stars may also form in AGN accretion disks due to fragmentation in the outer edges of the disk. While it is apparent that accretion disks around galactic sources exhibit instabilities, it is currently unclear if and how these instabilities operate in AGN, as the time scales involved are too long to observe transitions between quiescence and outburst. Furthermore, theoretical predictions of lightcurves based on these instabilities vary, and the existence and importance of disk stabilities can depend on how the viscosity is parameterized (e.g., Stella \& Rosner 1984; Szuszkiewicz 1990; Merloni 2003), how much of the accretion energy is dissipated in a hot corona (e.g., Svensson \& Zdziarski 1994), or if there is an outflow or an advection dominated accretion flow (ADAF) (e.g., Hameury et al. 2009).

The location in the disk where the instability occurs is important, as $p\left(L \mid \dot{M}, M_{B H}\right)$ is with respect to the optical flux in our model. Various instabilities are expected to operate in different regions of the disc, sometimes in a stochastic manner, and thus may or may not significantly affect the optical flux. The term $p\left(L \mid \dot{M}, M_{B H}\right)$ is also conditional on the source being seen as a BLQSO, and if the disk instabilities cause the ionizing continuum to disappear during quiescence, thus causing the broad emission lines to disappear as well, then $p\left(L \mid \dot{M}, M_{B H}\right)$ is only with regard to the distribution of optical luminosities during a disk outburst. Considering these issues, and the current uncertainty regarding the importance of disk instabilities, we consider it beyond scope of this paper to fully investigate the effects of time-dependent behavior in the accretion disk. However, in light of this discussion, disk instabilities can potentially have a significant effect on the distribution of luminosity at a given $M_{B H}$ (Siemiginowska \& Elvis 1997). Thus, the distribution of luminosity at a given $M_{B H}$ is likely altered beyond a simple power-law expected if the optical flux is trivially related to the external fueling rate.

In spite of these issues related to the detailed physics of the accretion disk, our estimated $p\left(L \mid M_{B H}\right)$ is qualitatively consistent with models which predict the BLQSO phase to occur while the quasar accretion rate is decaying, as we find that high Eddington ratio objects are rare, and that the number density of BLQSOs increases steeply toward lower values of $L / L_{E d d}$. In addition, although our estimated Eddington ratio distribution continues to rise toward lower $L / L_{E d d}$, our sample is too incomplete to rigorously probe the low Eddington ratio region of the distribution, and our estimated $L / L_{E d d}$ distribution in this region is heavily dependent on our parameteric form used to extrapolate beyond $L / L_{E d d} \lesssim 0.1$. Thus, we cannot test if the Eddington ratio distribution continues to rise until $L / L_{E d d} \sim 10^{-2}$, after which a steep decay in $p\left(L / L_{E d d}\right)$ might occur due to the disappearance of the broad emission lines (e.g. Churazov et al. 2005; Trump et al. 2009).

\subsection{Implications for the Growth of Supermassive Black Holes}

Our results in this work are broadly in agreement with recent observational and theoretical work suggesting that SMBH growth and spheroid formation have a common origin. In particular, models where mergers dominate black hole growth predict that major mergers of gas-rich galaxies initiate a burst of star formation (Mihos \& Hernquist 1994a, 1996), during which the black hole undergoes Eddington-limited obscured growth. Eventually the black hole becomes massive enough for radiation-driven feedback to unbind the surrounding gas, halting the accretion flow and revealing the object as a BLQSO (e.g., Hopkins et al. 2006b). As the activity further declines, the remnant will redden and become quiescent, satisfying the black hole-host galaxy correlation and leaving a dense stellar remnant from the starburst (Mihos \& Hernquist 1994b). This dense stellar remnant is identifiable as a second component in the light profiles of elliptical galaxies (Hopkins et al. 2008b, 2009c, d). Techniques based on the argument of Soltan (1982) have concluded that the SMBH accretion rate density of the Universe peaks at $z \sim 2$ (e.g., Marconi et al. 2004; Merloni \& Heinz 2008; Shankar et al. 2009), in agreement with the observed peak in the SMBH luminosity density of 
the Universe at $z \sim 2$ (e.g., Wolf et al. 2003; Hopkins et al. 2007b). We have found that the peak in the BLQSO cosmic mass density also occurs at $z \sim 2$, in broad agreement with models where the SMBH undergoes Eddington-limited growth up to the BLQSO phase, at least on a cosmological level.

In addition, our results suggest that a lower bound on the typical length of time for which a massive $\left(M_{B H} \sim 10^{9} M_{\odot}\right)$ SMBH could be seen as a BLQSO during a single BLQSO episode is $t_{B L} \gtrsim 120 \mathrm{Myr}$, i.e., a few Salpeter times. If all $M_{B H} \sim 10^{9} M_{\odot}$ SMBHs go through a BLQSO phase, and if the number density of SMBHs with $M_{B H} \sim 10^{9} M_{\odot}$ does not significantly increase from $z=1$ to $z=0$, then Equation (14) is no longer a lower bound and $t_{B L} \sim 150$ Myr represents an estimate of the lifetime of a single BLQSO phase. Indeed, if the SMBH's growth is self-regulated then there is good reason to assume that most SMBHs go through a BLQSO phase at the end of their growth (Hopkins \& Hernquist 2006), as the obscured/unobscured dichotomy represents an evolutionary sequence, at least at $z>1$. Furthermore, recent work employing the argument of Soltan (1982) suggests that the number density of $M_{B H} \sim 10^{9} M_{\odot}$ SMBHs does not significantly increase from $z=1$ to $z=0$ (Merloni \& Heinz 2008). These two considerations imply that $t_{B L} \sim 150 \mathrm{Myr}$ should represent a reasonable estimate of the length of the BLQSO phase.

Our estimated BLQSO lifetime of $t_{B L} \sim 150 \mathrm{Myr}$ is longer than the value of $t_{B L} \sim 10-20 \mathrm{Myr}$ predicted by Hopkins et al. (2006b). Part of this discrepancy may be due to the fact that they defined a BLQSO as having a B-band luminosity brighter than some fraction of the host galaxy's, while we define a BLQSO as simply having broad emission lines along our line of sight. Therefore, if a BLQSO has a decaying lightcurve, as assumed in the model of Hopkins et al. (2006b), then by our definition a SMBH may still be considered a BLQSO even after the B-band luminosity has fallen below its host's. This would imply that the predicted BLQSO lifetime under the definition of Hopkins et al. (2006b) would be shorter than our estimated value, as is the case. However, it is unclear if the lifetime of the BLQSO should be a factor of $\sim 10$ shorter under the definition of Hopkins et al. (2006b). Unfortunately, we cannot invert our estimated Eddington ratio distribution to a quasar lightcurve to test this, as the inversion is not unique, and we would have to assume a distribution of host galaxy B-band luminosity during the BLQSO phase at $z \sim 1$, which is poorly constrained; as Hopkins et al. (2006b) point out, uncertainty in the ratio of host galaxy to quasar B-band luminosity also introduces considerable systematic uncertainty in their predicted BLQSO lifetime. Moreover, our estimated lifetime is subject to the assumptions outlined in $\S 4.3$, which may introduce additional systematic uncertainties of a factor of a few. Considering this, a more quantitative comparison between our estimate and the prediction from Hopkins et al. (2006b) is difficult.

Our estimated BLQSO BHMF suggests a typical value of $M_{B H} \sim 10^{8} M_{\odot}$ for BLQSOs. Theoretical estimates of the mass distribution of SMBH seeds suggets that typical values for the seed mass should be $M_{B H} \sim 10^{5}-10^{6} M_{\odot}$ at $z \sim 10$ (Lodato \& Nataraian 2007; Pelupessy et al. 2007; Volonteri et al. 2008), and grow to $M_{B H} \sim 10^{8} M_{\odot}$ by $z \sim 3$ (Volonteri \& Natarajan 2009), consistent with our results. If the black hole's growth is Eddington-limited, then at least several Salpeter times are required to grow a seed black hole to $M_{B H} \sim 10^{9} M_{\odot}$ from a $M_{B H} \sim 10^{6} M_{\odot}$ seed, which is inconsistent with our estimated BLQSO lifetime. Furthermore, we find that most SMBHs in BLQSOs are not radiating near the Eddington limit, with a typical value being $L / L_{E d d} \sim 0.1$ at $M_{B H} \sim 10^{9} M_{\odot}$, as we might expect if BLQSOs are seen during a phase with a decaying fueling rate. Because most BLQSOs are radiating at considerably less than the Eddington limit, this therefore suggests that a factor of $\sim 10$ longer is needed to grow these SMBHs from seeds of $M_{B H} \sim 10^{6} M_{\odot}$, i.e., a growth time scale of $\sim 70$ Salpeter times. This corresponds to the age of the universe at $z \sim 2$, implying that sources observed at $z>2$ had to have been accreting at higher rates earlier in their growth.

An inferred growth time scale of $\sim 70$ Salpeter times is significantly longer than our estimated BLQSO lifetime at $z \sim 1$, implying one of a few explanations. First, it implies that if we assume that BLQSO SMBHs spend all of their growth as a BLQSO, then our assumption that all SMBHs go through a BLQSO phase along our line of sight is incorrect. In other words, this implies that some of the SMBH population that is growing at any redshift could never be observed by us to be a BLQSO at any time. Because the lifetime is related to what we can calculate from the BHMF by Equation (14), and if the SMBH spends all of its growth as a BLQSO, then an inferred SMBH growth time scale of $t_{B L} \sim 70 t_{\text {salp }}$ implies that only $\sim 4 \%$ of SMBHs ever go through a phase where we could observe them as BLQSOs at any time. This is an unrealistically low number, and thus we conclude that $t_{B L}$ is shorter than the growth time. Furthermore, it also illustrates that even if we can never observe a large fraction of active SMBHs, say $\sim 50-75 \%$, possibly due to orientation-dependent obscuration, then our estimated $t_{B L}$ is only underestimated by a factor of $\sim 2-4$.

Another possibility is that SMBHs in BLQSOs at $z \sim 1$ built up their mass via numerous fueling events of length $t_{B L} \gtrsim 150$ Myr. In this case one could grow a SMBH from a seed mass of $M_{B H} \sim 10^{6} M_{\odot}$ at $z \sim 10$, say, to a mass of $M_{B H} \sim 10^{9} M_{\odot}$ by $z \sim 1$, without the need for an earlier phase of obscured and accelerated growth. Similarly, we may have incorrectly assumed that the lifetime of the BLQSO phase is short compared to the time scale needed for any significant change in the BLQSO triggering time distribution, as this assumption was needed in order to use the observed distribution of BLQSOs as an estimate of their triggering distribution. This assumption may be incorrect if BLQSOs undergo a single long growth phase from $z \sim 10$ to $z \sim 1$. However, this possibility only exists for $z \lesssim 1.7$, since at higher redshifts the growth time for objects radiating at $L / L_{E d d} \sim 0.1$ is longer than the lookback time to $z \sim 10$, and thus BLQSOs that are observed at $z \gtrsim 1.7$ would have had to experience an earlier phase of obscured growth at an enhanced accretion rate. Moreover, if SMBHs that are seen as BLQSOs at $z \sim 1$ with $M_{B H} \sim 10^{9} M_{\odot}$ are grown in a single long BLQSO episode, or numerous repeated ones of $t_{B L} \sim 150 \mathrm{Myr}$, this would also imply that BLQSOs that are seen at $z \lesssim 1.7$ would have had a different fueling mechanism than BLQSOs that are seen at $z \gtrsim 1.7$, and it is unclear why this should be true. Therefore, we conclude that while part of the mass of SMBHs in 
BLQSOs may have been accumulated via multiple BLQSO episodes, it is unlikely that most of these SMBHs did not also experience a phase of obscured growth at an enhanced accretion rate.

If most SMBHs go through a BLQSO phase along our line of sight at some point in their growth, the fact that our estimated BLQSO lifetime is short compared to the SMBH growth time implies that SMBHs spend a significant amount of their time growing in a non-BLQSO phase, such as an obscured phase. Note that this argument is unaffected by the fact that at any $z$ we miss a considerable fraction of the SMBH population that is growing (e.g., due to obscuration), so long as these missed SMBHs undergo a BLQSO episode at some point in their life. The conclusion that a significant amount of growth occurs in an earlier obscured phase was also reached by Treister et al. (2010), and is expected from self-regulation models models where mergers or other triggering mechanisms fuel and initiate Eddington-limited accretion and obscured SMBH growth, until the SMBH becomes massive enough to unbind the ambient gas, revealing it as a BLQSO for $t_{B L} \sim 150 \mathrm{Myr}$. Within this interpretation, the BLQSO black hole mass density shown in Figure 6 is proportional to the rate at which the relic $\mathrm{SMBH}$ mass increases as a function of redshift. Furthermore, considering that the growth time scale for a SMBH radiating at $L / L_{E d d} \sim 0.1$ to grow from $10^{6} M_{\odot}$ to $10^{9} M_{\odot}$ corresponds to the age of the universe at $z \sim 2$, we also conclude that SMBHs accrete at a significantly higher rate during the earlier obscured phase, as compared to the BLQSO phase.

In this work we have found that the most massive SMBH that could be seen as a $\mathrm{BLQSO}$ is $M_{B H}^{M a x} \sim 3 \times 10^{10} M_{\odot}$ and would most likely be observed at $z \gtrsim 2$. These constraints on $M_{B H}^{M a x}$ are consistent with recent cosmological simulations of SMBH growth, as well as expectations from black hole feedback models. Cosmological simulations that follow the growth of SMBHs in bright $z \sim 6$ quasars have been able to grow SMBHs to $M_{B H} \sim 10^{9} M_{\odot}$ by $z \sim 4$ (Li et al. 2007; Di Matteo et al. 2008) and $M_{B H} \sim 2 \times 10^{10} M_{\odot}$ by $z \sim 2$ (Sijacki et al. 2009). Similarly, considerations based on quasar feedback and self-regulated SMBH growth, combined with the local distribution of bulge velocity dispersion, also suggest a value of $M_{B H}^{M a x} \sim 10^{10} M_{\odot}$ (Natarajan \& Treister 2009$)$.

If the SMBHs growth is self-regulated, then the final mass of the SMBH after a fueling event is set by the binding energy of the bulge regardless of the fueling mechanism (Younger et al. 2008). In order to buildup a mass as large as $M_{B H}^{M a x}$, multiple fueling episodes would likely be necessary, as is seen in cosmological simulations (e.g., Li et al. 2007; Di Matteo et al. 2008; Sijacki et al. 2009). However, it is unlikely that $M_{B H}^{M a x}$ could be increased significantly beyond the observed value as additional fueling mechanisms would likely result in only a small increase in the binding energy of the bulge. Therefore, additional fueling episodes would immediately result in the SMBH unbinding the accreting gas, preventing significant additional growth. Indeed, if our estimated BLQSO lifetime of $t_{B L} \sim 150$ Myr is correct for SMBHs with $M_{B H} \sim 10^{9} M_{\odot}$, and if the $\mathrm{SMBH}$ is radiating at a typical Eddington ratio of $L / L_{E d d} \sim 0.1$ during the BLQSO phase, then it would only accrete an additional $\sim 10^{8} M_{\odot}$, assuming a radiative efficiency of $\epsilon_{r}=0.1$. If the distribution of Eddington ratios is a power-law with $p\left(\Gamma_{E d d}\right) \propto \Gamma_{E d d}^{-1.5}$, as suggested by the discussion in $\S 5.1 .1$, then the typical Eddington ratio would be $L / L_{E d d} \lesssim 0.1$, suggesting even less growth during the BLQSO phase. Moreover, additional growth via black hole mergers, as might be expected from 'dry' mergers of galaxies, is also unlikely to lead to significant additional growth, as the mass of the additional SMBH is likely to be negligible compared to $M_{B H}^{M a x}$. And finally, additional growth through radiatively inefficient low-accretion rate modes does not contribute significantly to the black hole's final mass (Hopkins et al. 2006c; Cao 2007). Therefore, our estimated value of $M_{B H}^{M a x} \sim 3 \times 10^{10} M_{\odot}$ should be representative of the most massive SMBH for both active and inactive SMBHs.

Our results are consistent with previous data-based work that has attempted to map the distribution and growth of SMBHs. Most previous observational work has mapped SMBH growth by using the $M_{B H^{-}} \sigma$ relationship to infer the local BHMF, and then stepped backward in time using the quasar luminosity function to infer the contribution to the BHMF from accretion (e.g., Soltan 1982; Yu \& Tremaine 2002; Shankar et al. 2004; Marconi et al. 2004; Merloni \& Heinz 2008). In addition, there have been attempts to predict the BHMF and its evolution for all SMBHs, or all active SMBHs (e.g., Volonteri et al. 2003; Sijacki et al. 2007; Hopkins et al. 2008a; Di Matteo et al. 2008; Sijacki et al. 2009; Shen 2009). While not directly comparable to these studies, as we focus on broad line quasars, our results and conclusions are qualitatively consistent with previous observational and theoretical work in that we find evidence for self-regulated SMBH growth, black hole downsizing, and BLQSO lifetimes of $t_{B L} \sim 150 \mathrm{Myr}$.

\section{SUMMARY}

Our main results are:

- We have, for the first time, obtained an estimate of the black hole mass function for broad-line quasars that self-consistently corrects for incompleteness and the statistical uncertainty in the mass estimates derived from the broad emission lines in a statistically rigorous manner. Our estimated BHMF was obtained using data from the SDSS DR3 quasar sample.

- The standard deviation in the statistical error of the broad line mass estimates is less than the commonly used $\sim 0.4$ dex within the range of luminosity and redshift probed in our analysis. This may be due to correlation between the error in the mass estimates and luminosity and/or redshift, or a dependence of the standard deviation of the error on luminosity and/or redshift. When we treat the standard deviation in the statistical error as a free parameter, we estimate that the the $\mathrm{Mg}$ II-based mass estimates scatter about the reverberation mapping estimates with an amplitude of $\approx 0.18 \mathrm{dex}$, while the $\mathrm{C}$ IV-based estimate scatter with an amplitude of $\approx 0.13$ dex. 
- We find evidence for cosmic downsizing among BLQSOs, where the number density of BLQSOs peaks at higher redshift with increasing black hole mass.

- We find that the comoving mass density of SMBHs in BLQSOs peaks at $z \sim 2$. We use our estimate for the BHMF to place constraints on the duty cycle, $\delta\left(M_{B H}, z\right)$, and lifetime, $t_{B L}$, for BLQSOs. The duty cycle at $z=1$ is constrained to be $\delta \gtrsim 0.01$ at $M_{B H} \sim 10^{9} M_{\odot}$, falling to $\delta \gtrsim 10^{-5}$ at $M_{B H} \sim 10^{10} M_{\odot}$. We estimate the lifetime of the BLQSO phase for SMBHs of $M_{B H}=10^{9} M_{\odot}$ at $z=1$ to be $t_{B L}=150 \pm 15 \mathrm{Myr}$. However, we will have underestimated the BLQSO lifetime if there is a population of $M_{B H}=10^{9} M_{\odot}$ SMBHs that never experience a BLQSO phase along our line of sight, or if the local number density of these black holes is significantly larger than the $z=1$ number density of these black holes. We argue that our estimated BLQSO lifetime, in combination with the estimated Eddington ratio distribution, suggests that most of a SMBH's growth occurs when it is not seen as a BLQSO and accreting at a higher rate, and that BLQSO activity represents a short phase that most SMBHs go through, consistent with self-regulated growth models.

- We estimate that the most massive SMBH that could be seen as a BLQSO is $M_{B H} \approx 3 \times 10^{10} M_{\odot}$. This SMBH would most likely be seen as a BLQSO at $z>2$. While largely in agreement with previous work, we have for the first time obtained statistically rigorous constraints on the value of $M_{B H}^{M a x}$ and its redshift.

- Assuming a constant bolometric correction of $C_{1350}=4.3$ (Vestergaard \& Osmer 2009), our inferred distribution of Eddington ratios peaks at $L / L_{E d d} \sim 0.05$ and has a dispersion of $\sim 0.4$ dex. Compared to previous work, our inferred Eddington ratio distribution is broader and shifted toward lower values of $L / L_{E d d}$, showing that previous estimated distributions of $L / L_{E d d}$ were significantly affected by incompleteness. We therefore provide evidence that most BLQSOs are not radiating at or near the Eddington limit, and that there is a large dispersion in Eddington ratio for BLQSOs. In addition, we also find that the number density of BLQSOs increases steeply toward lower values of $L / L_{E d d}$, consistent with models where the BLQSO phase occurs when the fuel supply is dwindling or halted.

- The evolution of the cosmological SMBH mass density for BLQSOs tracks the evolution in the cosmological accretion rate density of SMBHs estimated from variations of the Soltan (1982) argument (Marconi et al. 2004; Merloni \& Heinz 2008; Shankar et al. 2009). This result, in combination with our estimated BLQSO lifetime and Eddington ratio distribution, are qualitatively consistent with models of self-regulated SMBH growth, with the BLQSO phase occuring at the end of the SMBHs obscured Eddington-limited growth (e.g., Hopkins \& Hernquist 2006; Hopkins et al. 2006b).

We thank Yue Shen, Priyamvada Natarajan, Martin Elvis, and Charles Steinhardt for helpful discussions and comments on this paper, Mark Ammons and Aleks Diamond-Stanic for helpful discussions, and the anonymous referee for a careful reading and comments that lead to improvement of this work. BK acknowledges support by NASA through Hubble Fellowship grants \#HF-01220.01 and \#HF-51243.01 awarded by the Space Telescope Science Institute, which is operated by the Association of Universities for Research in Astronomy, Inc., for NASA, under contract NAS 5-26555. MV acknowledges financial support through grants HST-AR-10691, HST-GO-10417, and HST-GO-10833 from NASA through the Space Telescope Science Institute, which is operated by the Association of Universities for Research in Astronomy, Inc., under NASA contract NAS5-26555. XF acknowledges support by NSF grant AST-0806861, a Packard Fellowship for Science and Engineering, a John Simon Guggenheim Memorial Fellowship and the Max Planck Society. The Dark Cosmology Centre is funded by the Danish National Research Foundation.

\section{REFERENCES}

Akritas, M. G., \& Bershady, M. A. 1996, ApJ, 470, 706

Aller, M. C., \& Richstone, D. O. 2007, ApJ, 665, 120

Begelman, M. C., \& Nath, B. B. 2005, MNRAS, 361, 1387

Booth, C. M., \& Schaye, J. 2009, in press at MNRAS(arXiv:0904.2572)

Bower, R. G., Benson, A. J., Malbon, R., Helly, J. C., Frenk, C. S., Baugh, C. M., Cole, S., \& Lacey, C. G. 2006, MNRAS, 370, 645

Bruzual, G., \& Charlot, S. 2003, MNRAS, 344, 1000

Cannizzo, J. K., Lee, H. M., \& Goodman, J. 1990, ApJ, 351, 38

Cao, X. 2007, ApJ, 659, 950

Cattaneo, A., Blaizot, J., Devriendt, J., \& Guiderdoni, B. 2005, MNRAS, 364, 407

Churazov, E., Sazonov, S., Sunyaev, R., Forman, W., Jones, C., Bohringer, H. 2005, MNRAS, 363, L91

Ciotti, L., \& Ostriker, J. P. 2001, ApJ, 551, 131

Constantin, A., Green, P., Aldcroft, T., Kim, D.-W., Haggard, D., Barkhouse, W., \& Anderson, S. F. 2009, in press at ApJ (arXiv:0909.4086)
Croom, S. M., Smith, R. J., Boyle, B. J., Shanks, T., Miller, L., Outram, P. J., \& Loaring, N. S. 2004, MNRAS, 349, 1397

Croton, D. J., et al. 2006, MNRAS, 365, 11

Croton, D. J. 2009, MNRAS, 394, 1109

Czerny, B., Siemiginowska, A., Janiuk, A., Nikiel-Wroczynski, B., \& Stawarz, L. 2009, in press at ApJ (arXiv:0903.3940)

Denney, K. D., Peterson, B. M., Dietrich, M., Vestergaard, M., \& Bentz, M. C. 2009, ApJ, 692, 246

Di Matteo, T., Colberg, J., Springel, V., Hernquist, L., \& Sijacki, D. 2008, ApJ, 676, 33

Di Matteo, T., Springel, V., \& Hernquist, L. 2005, Nature, 433, 604

Fabian, A. C. 1999, MNRAS, 308, L39

Fan, X., et al. 2001a, AJ, 121, 31

Fan, X., et al. 2001b, AJ, 122, 2833

Fine, S., et al. 2008, MNRAS, 390, 1413

Gabor, J. M., et al. 2009, ApJ, 691, 705

Gavignaud, I., et al. 2008, A\&A, 492, 637

Gebhardt, K., et al. 2000, ApJ, 539, L13 
Gelman, A., Carlin, J. B., Stern, H. S., \& Rubin, D. B. 2004, Bayesian Data Analysis (2nd ed.; Boca Raton:Chapman \& Hall/CRC)

Georgakakis, A., et al. 2009, in press at MNRAS(arXiv:0904.3747)

Gonçalves, T. S., Steidel, C. C., \& Pettini, M. 2008, ApJ, 676, 816

Goodman, J., \& Tan, J. C. 2004, ApJ, 608, 108

Graham, A. W., \& Driver, S. P. 2007, ApJ, 655, 77

Graham, A. W., Erwin, P., Caon, N., \& Trujillo, I. 2001, ApJ, 563, L11

Greene, J. E., \& Ho, L. C. 2006, ApJ, 641, L21

Greene, J. E., \& Ho, L. C. 2007, ApJ, 667, 131

Guyon, O., Sanders, D. B., \& Stockton, A. 2006, ApJS, 166, 89

Haehnelt, M. G., \& Kauffmann, G. 2000, MNRAS, 318, L35

Haehnelt, M. G., Natarajan, P., \& Rees, M. J. 1998, MNRAS, 300,817

Hameury, J.-M., Viallet, M., \& Lasota, J.-P. 2009, A\&A, 496, 413

Häring, N., \& Rix, H.-W. 2004, ApJ, 604, L89

Hasinger, G., Miyaji, T., \& Schmidt, M. 2005, A\&A, 441, 417

Hatziminaoglou, E., Siemiginowska, A., \& Elvis, M. 2001, ApJ, 547,90

Heckman, T. M., Kauffmann, G., Brinchmann, J., Charlot, S., Tremonti, C., \& White, S. D. M. 2004, ApJ, 613, 109

Hickox, R. C., et al. 2009, ApJ, 696, 891

Hirose, S., Krolik, J. H., \& Blaes, O. 2008, in press at ApJ (arXiv:0809.1708)

Hopkins, P. F., \& Hernquist, L. 2006, ApJS, 166, 1

Hopkins, P. F., \& Hernquist, L. 2009, ApJ, 698, 1550

Hopkins, P. F., Hernquist, L., Cox, T. J., Di Matteo, T., Martini, P., Robertson, B., \& Springel, V. 2005a, ApJ, 630, 705

Hopkins, P. F., Hernquist, L., Cox, T. J., Di Matteo, T., Robertson, B., \& Springel, V. 2005b, ApJ, 630, 716

Hopkins, P. F., Hernquist, L., Cox, T. J., Di Matteo, T., Robertson, B., \& Springel, V. 2005c, ApJ, 632, 81

Hopkins, P. F., Hernquist, L., Cox, T. J., Robertson, B., Di Matteo, T., \& Springel, V. 2006a, ApJ, 639, 700

Hopkins, P. F., Hernquist, L., Cox, T. J., Di Matteo, T., Robertson, B., \& Springel, V. 2006b, ApJS, 163, 1

Hopkins, P. F., Hernquist, L., Cox, T. J., \& Kereš, D. 2008a, ApJS, 175, 356

Hopkins, P. F., Hernquist, L., Cox, T. J., Robertson, B., \& Krause, E. 2007a, ApJ, 669, 67

Hopkins, P. F., Hernquist, L., Cox, T. J., Robertson, B., \& Krause, E. 2007c, ApJ, 669, 45

Hopkins, P. F., Hernquist, L., Cox, T. J., Dutta, S. N., \& Rothberg, B. 2008b, ApJ, 679, 156

Hopkins, P. F., Cox, T. J., Dutta, S. N., Hernquist, L., Kormendy, J., \& Lauer, T. R. 2009c, ApJS, 181, 135

Hopkins, P. F., Lauer, T. R., Cox, T. J., Hernquist, L., \& Kormendy, J. 2009d, ApJS, 181, 486

Hopkins, P. F., Hickox, R., Quataert, E., \& Hernquist, L. 2009a, submitted to MNRAS (arXiv:0901.2936)

Hopkins, P. F., Murray, N., \& Thompson, T. 2009b, submitted to MNRAS (arXiv:0903.3949)

Hopkins, P. F., Narayan, R., \& Hernquist, L. 2006c, ApJ, 643, 641

Hopkins, P. F., Richards, G. T., \& Hernquist, L. 2007b, ApJ, 654, 731

Janiuk, A., Czerny, B., \& Siemiginowska, A. 2000, ApJ, 542, L33

Janiuk, A., Czerny, B., Siemiginowska, A., \& Szczerba, R. 2004, ApJ, 602, 595

Jiang, L., Fan, X., Vestergaard, M., Kurk, J. D., Walter, F., Kelly, B. C., \& Strauss, M. A. 2007, AJ, 134, 1150

Johansson, P. H., Naab, T., \& Burkert, A. 2009, ApJ, 690, 802

Kaspi, S., Brandt, W. N., Maoz, D., Netzer, H., Schneider, D. P., \& Shemmer, O. 2007, ApJ, 659, 997

Kauffmann, G., \& Haehnelt, M. 2000, MNRAS, 311, 576 \& Strauss, M. A. 2007, AJ, 134, 1150

Kelly, B. C. 2007, ApJ, 665, 1489

Kelly, B. C., \& Bechtold, J. 2007, ApJS, 168, 1

Kelly, B. C., Bechtold, J., \& Siemiginowska, A. 2009a, ApJ, 698, 895

Kelly, B. C., Bechtold, J., Trump, J. R., Vestergaard, M., \& Siemiginowska, A. 2008, ApJS, 176, 355

Kelly, B. C., Vestergaard, M., \& Fan, X. 2009b, ApJ, 692, 1388 (KVF09)

King, A. R., \& Pringle, J. E. 2007, MNRAS, 377, L25

Kollmeier, J. A., et al. 2006, ApJ, 648, 128

Kormendy, J., \& Richstone, D. 1995, ARA\&A, 33, 581
Kozłowski, S., et al. 2010, ApJ, 708, 927

Krolik, J. H. 2001, ApJ, 551, 72

La Franca, F., et al. 2005, ApJ, 635, 864

Labita, M., Decarli, R., Treves, A., \& Falomo, R. 2009a, MNRAS, 396,1537

Labita, M., Decarli, R., Treves, A., \& Falomo, R. 2009b, in press at MNRAS(arXiv:0907.2963)

Lasota, J.-P. 2001, New Astronomy Review, 45, 449

Lauer, T. R., et al. 2007, ApJ, 662, 808

Li, Y., et al. 2007, ApJ, 665, 187

Lin, D. N. C., \& Shields, G. A. 1986, ApJ, 305, 28

Liu, J. S. 2004, Monte Carlo Strategies in Scientific Computing (New York:Springer)

Lodato, G., \& Natarajan, P. 2007, MNRAS, 377, L64

Magorrian, J., et al. 1998, AJ, 115, 2285

Marconi, A., Axon, D. J., Maiolino, R., Nagao, T., Pastorini, G.,

Pietrini, P., Robinson, A., \& Torricelli, G. 2008, ApJ, 678, 693

Marconi, A., \& Hunt, L. K. 2003, ApJ, 589, L21

Marconi, A., Risaliti, G., Gilli, R., Hunt, L. K., Maiolino, R., \&

Salvati, M. 2004, MNRAS, 351, 169

Martini, P. 2004, Coevolution of Black Holes and Galaxies, 169

Marulli, F., Branchini, E., Moscardini, L., \& Volonteri, M. 2007, MNRAS, 375, 649

McLure, R. J., \& Dunlop, J. S. 2001, MNRAS, 327, 199

McLure, R. J., \& Dunlop, J. S. 2002, MNRAS, 331, 795

McLure, R. J., \& Dunlop, J. S. 2004, MNRAS, 352, 1390

Menou, K., \& Quataert, E. 2001, ApJ, 552, 204

Merloni, A. 2003, MNRAS, 341, 1051

Merloni, A. 2004, MNRAS, 353, 1035

Merloni, A., \& Heinz, S. 2008, MNRAS, 388, 1011

Merloni, A., et al. 2010, ApJ, 708, 137

Merritt, D., \& Ferrarese, L. 2001, ApJ, 547, 140

Metzroth, K. G., Onken, C. A., \& Peterson, B. M. 2006, ApJ, 647,901

Mihos, J. C., \& Hernquist, L. 1994a, ApJ, 437, L47

Mihos, J. C., \& Hernquist, L. 1994b, ApJ, 437, 611

Mihos, J. C., \& Hernquist, L. 1996, ApJ, 464, 641

Murray, N., Quataert, E., \& Thompson, T. A. 2005, ApJ, 618, 569

Natarajan, P., \& Treister, E. 2009, MNRAS, 393, 838

Nayakshin, S., Rappaport, S., \& Melia, F. 2000, ApJ, 535, 798

Netzer, H., Lira, P., Trakhtenbrot, B., Shemmer, O., \& Cury, I. 2007, ApJ, 671, 1256

Netzer, H., \& Trakhtenbrot, B. 2007, ApJ, 654, 754

Pelupessy, F. I., Di Matteo, T., \& Ciardi, B. 2007, ApJ, 665, 107

Pringle, J. E. 1991, MNRAS, 248, 754

Richards, G. T., et al. 2006, AJ, 131, 2766

Salucci, P., Szuszkiewicz, E., Monaco, P., \& Danese, L. 1999, MNRAS, 307, 637

Scoville, N., et al. 2007, ApJS, 172, 1

Shakura, N. I., \& Sunyaev, R. A. 1973, A\&A, 24, 337

Shankar, F., Salucci, P., Granato, G. L., De Zotti, G., \& Danese, L. 2004, MNRAS, 354, 1020

Shankar, F., Weinberg, D. H., \& Miralda-Escudé, J. 2009, ApJ, 690, 20

Shen, Y., Greene, J. E., Strauss, M. A., Richards, G. T., \& Schneider, D. P. 2008, ApJ, 680, 169

Shen, Y. 2009, ApJ, 704, 89

Shen, Y., \& Kelly, B. C. 2010, ApJ, 713, 41

Siemiginowska, A., Czerny, B., \& Kostyunin, V. 1996, ApJ, 458, 491

Siemiginowska, A., \& Elvis, M. 1997, ApJ, 482, L9

Sijacki, D., Springel, V., di Matteo, T., \& Hernquist, L. 2007, MNRAS, 380, 877

Sijacki, D., Springel, V., \& Haehnelt, M. G. 2009, submitted to MNRAS(arXiv:0905.1689)

Silk, J., \& Rees, M. J. 1998, A\&A, 331, L1

Silverman, J. D., et al. 2008, ApJ, 679, 118

Small, T. A., \& Blandford, R. D. 1992, MNRAS, 259, 725

Soltan, A. 1982, MNRAS, 200, 115

Somerville, R. S., Hopkins, P. F., Cox, T. J., Robertson, B. E., \& Hernquist, L. 2008, MNRAS, 391, 481

Springel, V., Di Matteo, T., \& Hernquist, L. 2005, ApJ, 620, L79

Steffen, A. T., Barger, A. J., Cowie, L. L., Mushotzky, R. F., \&

Yang, Y. 2003, ApJ, 596, L23

Steinhardt, C. L., \& Elvis, M. 2010, MNRAS, 402, 2637

Steinhardt, C. L., \& Elvis, M. 2010, in press at

MNRAS (arXiv:0912.0734) 
Stella, L., \& Rosner, R. 1984, ApJ, 277, 312

Svensson, R., \& Zdziarski, A. A. 1994, ApJ, 436, 599

Szuszkiewicz, E. 1990, MNRAS, 244, 377

Treister, E., Natarajan, P., Sanders, D., Urry, C. M., Schawinski,

K., \& Kartaltepe, J. 2010, in press at Science (arXiv:1003.4736

Tremaine, S., et al. 2002, ApJ, 574, 740

Trump, J. R., et al. 2007, ApJS, 172, 383

Trump, J. R., et al. 2009, in press at ApJ (arXiv:0905.1123)

Ueda, Y., Akiyama, M., Ohta, K., \& Miyaji, T. 2003, ApJ, 598, 886

Ulrich, M.-H., Maraschi, L., \& Urry, C. M. 1997, ARA\&A, 35, 445

Vasudevan, R. V., \& Fabian, A. C. 2007, MNRAS, 381, 1235

Véron-Cetty, M.-P., Joly, M., \& Véron, P. 2004, A\&A, 417, 515

Vestergaard, M. 2004, ApJ, 601, 676

Vestergaard, M. 2010, Proceedings of 2007 STScI Spring Symposium "Black Holes", Cambridge University Press, in press (arXiv:0904.2615)

Vestergaard, M., Fan, X., Tremonti, C. A., Osmer, P. S., \& Richards, G. T. 2008, ApJ, 674, L1
Vestergaard, M., \& Osmer, P. 2009, in press at ApJ(arXiv:0904:3348)

Vestergaard, M., \& Peterson, B. M. 2006, ApJ, 641, 689

Vestergaard, M., \& Wilkes, B. J. 2001, ApJS, 134, 1

Volonteri, M., Haardt, F., \& Madau, P. 2003, ApJ, 582, 559

Volonteri, M. 2007, ApJ, 663, L5

Volonteri, M., Lodato, G., \& Natarajan, P. 2008, MNRAS, 383, 1079

Volonteri, M., \& Natarajan, P. 2009, MNRAS, 400, 1911

Wang, J.-M., Chen, Y.-M., \& Zhang, F. 2006, ApJ, 647, L17

Wolf, C., Wisotzki, L., Borch, A., Dye, S., Kleinheinrich, M., \& Meisenheimer, K. 2003, A\&A, 408, 499

Wyithe, J. S. B., \& Loeb, A. 2003, ApJ, 595, 614

Young, M., Elvis, M., \& Risaliti, G. 2010, ApJ, 708, 1388

Younger, J. D., Hopkins, P. F., Cox, T. J., \& Hernquist, L. 2008, ApJ, 686, 815

Yu, Q., \& Lu, Y. 2004, ApJ, 602, 603

Yu, Q., \& Lu, Y. 2008, ApJ, 689, 732

Yu, Q., Lu, Y., \& Kauffmann, G. 2005, ApJ, 634, 901

Yu, Q., \& Tremaine, S. 2002, MNRAS, 335, 965 